\title{
Effects of modality on subjective frequency estimates and processing of spoken and printed words
}

\author{
DANIEL E. GAYGEN and PAUL A. LUCE \\ State University of New York, Buffalo, New York
}

\begin{abstract}
Previous research (Garber \& Pisoni, 1991; Pisoni \& Garber, 1990) has demonstrated that subjective familiarity judgments for words are not differentially affected by the modality (visual or auditory) in which the words are presented, suggesting that participants base their judgments on fairly abstract, modality-independent representations in memory. However, in a recent large-scale study in Japanese (Amano, Kondo, \& Kakehi, 1995), marked modality effects on familiarity ratings were observed. The present research further examined possible modality differences in subjective ratings and their implications for word recognition. Specially selected words were presented to participants for frequency judgments. In particular, participants were asked how frequently they read, wrote, heard, or said a given spoken or printed word. These ratings were then regressed against processing times in auditory and visual lexical decision and naming tasks. Our results suggest modality dependence for some lexical representations.
\end{abstract}

The effects of word frequency are ubiquitous in research on visual and spoken word recognition (Forster \& Chambers, 1973; Luce, Pisoni, \& Goldinger, 1990), and considerable modeling efforts have been devoted to explaining the mechanisms responsible for the findings that higher frequency words are recognized more quickly and accurately than are lower frequency words. Typically, frequencies of stimuli have been estimated from counts of printed material (e.g., Kučera \& Francis, 1967; Thorndike \& Lorge, 1944). Recently, however, subjective ratings of word frequency have provided an alternative measure for investigating effects of frequency on recognition. To obtain subjective ratings, participants are asked directly how familiar they are with individually presented words. Gernsbacher (1984) has shown that many previous inconsistencies in research on word recognition can be dispelled when objective frequency counts are supplanted by subjective ratings.

More recently, researchers have used subjective familiarity ratings to examine the degree to which lexical representations that support word recognition are independent of modality of processing (i.e., visual or auditory). If subjective ratings are modality independent, ratings for words presented visually should not differ from ratings

This research was supported (in part) by Research Grant 1 R0I DC 0265801-A 1 from the National Institute on Deafness and Other Communication Disorders, National Institutes of Health. The authors would like to thank Peter Jusczyk, Jim Sawsuch, Gail Mauner, and three anonymous reviewers for their comments, and Lorrie Chappell and Jennifer Duchnick for their assistance in running participants. Correspondence should be addressed to either author at the Language Perception Laboratory, Department of Psychology, State University of New York, Buffalo, NY 14260 (e-mail: dan@deuro.fss.buffalo.edu, or paul@deuro. fss.buffalo.edu). for words presented auditorily. On the other hand, modality dependence of lexical representations should be revealed by differential judgments of words that are processed in the visual or auditory modalities. If participants' mental representations of visual and spoken words incorporate possible differences in frequency of processing in the two modalities, subjective ratings should reveal these differences. In short, modality dependence should be reflected in lower cross-modality correlations between subjective ratings of words than within-modality correlations. Conversely, modality-independent lexical representations should not result in differentially sensitive ratings.

Gernsbacher (1984), Connine, Mullennix, Shernoff, and Yelen (1990), Pisoni and Garber (1990), and Garber and Pisoni (1991), among others, have argued that subjective familiarity ratings of word frequency are modality independent. They found high correlations between subjective ratings for words presented visually and the same words presented auditorily. However, subsequent research on subjective ratings has suggested that the mental representations of the frequency of lexical items are not modality independent. In a large-scale study of Japanese, Amano, Kondo, and Kakehi (1995) obtained correlations between ratings for visually and auditorily presented words that were much lower than those reported by Pisoni and Garber, suggesting a lack of modality independence for information in memory regarding familiarity.

One major difference between the Pisoni and Garber (1990) study and the Amano et al. (1995) study is that Amano et al. used a stimulus set nearly 70 times the size of Pisoni and Garber's list. Consequently, Amano et al.'s list contained a much higher proportion of low-frequency words. The lower cross-modality correlations observed by Amano et al. may have been due to the greater repre- 
sentation of low-frequency words in their stimulus list. Amano et al. also found that correlations between ratings for low-frequency words were significantly lower than correlations between ratings for medium-frequency words, which were, in turn, lower than correlations between ratings for high-frequency words. These results suggest that modality dependence may vary as a function of word frequency, with low-frequency words showing the strongest effects of modality-specific processing.

Early versions of two models of word recognition have made at least implicit claims regarding the modality dependence of frequency information. Although Morton's (1969) original logogen model was consistent with modality independence, later versions explicitly acknowledged modality dependence by incorporating separate logogen systems for printed and spoken words (Morton, 1979). In addition, Forster's (1976) autonomous search model is consistent with modality dependence, because the first stage of the model involves submitting an unanalyzed pattern to peripheral access files made up of bins that contain either frequency-ordered orthographic entries or frequency-ordered phonetic entries. Thus, in Forster's model, frequency effects should depend directly on modality of processing.

More recent models of word recognition in the connectionist tradition have yet to address specifically the issue of modality dependence of frequency indices in memory. For example, the interactive-activation model of visual word recognition (McClelland \& Rumelhart, 1981) encodes frequency only at the level of fairly abstract lexical nodes, which may or may not be modality independent. If frequency indices are dependent on modality-specific, form-based representations, a complete connectionist account of lexical processing may need to encode frequency at levels of representation below that of more abstract lexical nodes.

The present research further examined whether word frequency is a modality-independent phenomenon. In particular, we attempted to determine whether frequency information is coded strictly at an abstract lexical level or whether this information is also present at one or more particular, form-based levels of representation, such as phonetic and orthographic representations that support perception and production. This research attempted to extend previous findings to determine whether formbased, modality-dependent representations possess their own frequency indices. Moreover, the present research explicitly examined words at various frequency levels to assess the possibility that the magnitude of modalitydependence effects vary as a function of frequency (see Amano et al., 1995).

We examined subjective frequency ratings for the four major forms of word usage: hearing, speaking, reading, and writing. Note that these forms vary on sensory channel (visual and auditory) and performance (perception and production) dimensions. Because words may be en- countered in each of these forms at varying frequencies, frequency indices for each of the forms in memory may differ. If, on the other hand, lexical entries contain only abstract frequency information that is independent of form frequency, indices in memory should be unitary phenomena incrementing equally with each encounter with a word, regardless of form. (We assume that objective and subjective measures of frequency and familiarity are based on essentially the same type of information stored in memory. However, see below for a more detailed treatment of this issue.)

We gathered subjective frequency ratings for 252 words using the following questions: "How often have you read the word __ in your lifetime?" "How often have you written the word _ in your lifetime?" "How often have you heard the word __ in your lifetime?" and "How often have you said the word _ in your lifetime?" Separate ratings were collected for a list of words presented visually and the same list of words presented auditorily.

We will refer to three important terminological distinctions throughout this paper: The first distinction, modality, refers to stimulus presentation format. Words were presented for ratings either visually or auditorily. The second and third distinctions refer to the types of questions on which the participants based their ratings: Sensory channel refers to questions regarding eye (read and write) versus ear (hear and say). Performance refers to questions regarding perception (read and hear) versus production (write and say).

We were interested not only in the ratings themselves but also in their implications for processing times. In particular, we examined the degree to which each of the four types of ratings correlate with latencies in processing tasks. We attempted to determine whether subjective ratings of the frequencies of producing words (writing, saying) correlate with latencies in experiments with a production component (naming), whether subjective ratings of frequencies of perceiving words (reading, hearing) correlate with latencies in experiments with perception components (lexical decision), and whether ratings of a given input modality correlate with latencies within and across modalities. Again, we were interested in determining whether frequency differences are coded strictly at an abstract lexical level or whether this information is also present at one or more particular, form-based levels of representation. We were also interested in examining these effects at various levels of frequency.

We performed two experiments to investigate these questions. In Experiment 1, participants rated how frequently they read, wrote, heard, and said the stimulus words. Visually presented words and auditorily presented words were rated separately. In Experiment 2, we examined processing time and accuracy in four word recognition paradigms for the words rated in Experiment 1. These paradigms were (1) auditory lexical decision, (2) visual lexical decision, (3) auditory repetition, and (4) visual naming. 


\section{EXPERIMENT 1A \\ Subjective Frequency Ratings for Auditorily Presented Words}

\section{Method}

\section{Participants}

Forty undergraduates at the State University of New York at Buffalo participated in partial fulfillment of requirements for an introductory psychology course. All participants were native speakers of English, were right-handed, and reported no history of speech, hearing, or visual disorders.

\section{Materials}

Three lists of 84 words each were chosen from an on-line lexicon based on Webster's (1967) dictionary. One list consisted of highfrequency words, one of medium-frequency words, and one of lowfrequency words. High-frequency words occurred 100 times or more per million printed words (Kučera \& Francis, 1967). Mediumfrequency words occurred from 6-99 times per million. Lowfrequency words occurred 5 or fewer times per million. Each list had equal numbers of one, two, and three syllable words.

Subjective familiarity ratings were also used to select the stimuli (Nusbaum, Pisoni, \& Davis, 1984). High-frequency words were rated 6 or above on a 7-point scale of familiarity $(1=$ don't know the word; $7=$ know the word and know its meaning). Mediumfrequency words were rated above 3 and below 6 on the same scale. Low-frequency words were rated 3 or below. These criteria were used to ensure that the lists contained words that were in the same frequency and familiarity category (i.e., no words were of low frequency but of high familiarity, etc.) and that words falling in the highest category occurred frequently and were known to the participants and those in the lowest category occurred rarely and were less familiar. The combined lists of stimulus words and their ratings can be found in the Appendix. ${ }^{1}$

The words were recorded by a male talker. To ensure maximum intelligibility when presented in isolation to participants, all words were also spoken in isolation. The stimuli were low-pass filtered at $4.8 \mathrm{kHz}$ and digitized at a sampling rate of $10 \mathrm{kHz}$ using a 12-bit analog-to-digital converter. All words were edited, placed into individual files, and stored on computer disk. Stimuli were output binaurally over matched and calibrated TDH-39 headphones.

\section{Procedure}

The participants were seated in individual booths equipped with a computer display and a keyboard. The stimuli were presented auditorily to the participants over headphones. Stimuli were played and responses collected by a PDP-11/34 computer. A row of asterisks presented on the computer display signaled the beginning of a new trial. On each trial, a word was played over the headphones, and the participants rated the frequency of the word on a 10-point scale ranging from 1 (never) to 10 (often). The participants' ratings were in response to one of four questions: "How often have you read the word ___ in your lifetime?" "How often have you heard the word __ in your lifetime?" "How often have you written the word __ in your lifetime?" or "How often have you said the word _ in your lifetime?"

The combined list of high-, medium-, and low-frequency words was divided into four blocks. The participants rated one block in the read condition, one block in the hear condition, one block in the write condition, and one block in the say condition. The order of block presentation was counterbalanced across participants. There were equal numbers of high-, medium-, and low-frequency words and one-, two-, and three-syllable words in each block. No single participant rated a given word in more than one condition. However, every individual word was rated in every condition. There were five judgments per item per condition. To summarize, (1) rat- ing condition was a within-participants factor, (2) no given word was rated more than once by an individual participant, and (3) all words were rated in all conditions.

\section{EXPERIMENT 1B \\ Subjective Frequency Ratings for Visually Presented Words}

\section{Method}

\section{Participants}

A different group of 40 undergraduates at the State University of New York at Buffalo participated in partial fulfillment of requirements for an introductory psychology course. All participants were native speakers of English, were right-handed, and reported no history of speech, hearing, or visual disorders.

\section{Materials}

The same words used in Experiment 1A were presented visually to the participants for subjective ratings of frequency.

\section{Procedure}

The participants were seated in individual booths equipped with a Microterm 5510 computer display and keyboard. A row of asterisks signaled the beginning of a trial, after which a target word was presented. The word remained on the screen until a rating was made. Stimuli were presented and responses collected by a PDP $11 / 34$ computer. All other procedures were the same as those in Experiment $1 \mathrm{~A}$.

\section{Results of Experiments $1 A$ and $1 B$}

A separate $4 \times 3$ [question (read, write, hear, say) $\times$ frequency (high, medium, low)] analysis of variance (ANOVA) was performed for each of the presentation types (visual, auditory). Table 1 shows mean ratings for each question for visually and auditorily presented words.

\section{Auditorily Presented Words}

For auditorily presented words, a significant main effect was obtained for frequency $[F(2,39)=965.60, p<$ .0001 ; mean rating for high $=7.6$; mean rating for medium $=3.7$; mean rating for low $=2.2$ ]. As expected, subjective ratings varied as a function of frequency of the stimulus items. Ratings were highest for high-frequency words and lowest for low-frequency words.

A significant main effect of question was also obtained $[F(3,39)=27.65, p<.0001$; mean rating for read $=4.7$; mean rating for write $=4.0$; mean rating for hear $=5.0$; mean rating for say $=4.3$ ]. To examine the overall main

Table 1

Mean Ratings for Each Question in Each Modality

\begin{tabular}{lllll}
\hline Modality & Read & Write & Hear & Say \\
\hline \multicolumn{5}{c}{ High-Frequency Words } \\
Visual & 8.42 & 7.93 & 8.44 & 8.20 \\
Auditory & 7.80 & 6.91 & 8.09 & 7.56 \\
& \multicolumn{5}{c}{ Medium-Frequency Words } \\
Visual & 4.02 & 3.32 & 3.93 & 3.27 \\
Auditory & 3.80 & 3.02 & 4.18 & 3.31 \\
\multicolumn{5}{c}{ Low-Frequency Words } \\
Visual & 1.99 & 1.55 & 1.86 & \\
Auditory & 2.34 & 2.08 & 2.53 & 2.43 \\
\hline
\end{tabular}


effect of question in detail, planned contrasts were performed to determine whether ratings for auditorily presented words differed as a function of the sensory channel (eye vs. ear) and performance (perception vs. production) dimensions. Collapsing across frequency, ear ratings (mean rating $=4.6$ ) were significantly higher than eye ratings (mean rating $=4.4)[F(1,39)=9.87, p<.0021]$, and perception ratings (mean rating $=4.8$ ) were significantly higher than production ratings (mean rating $=$ $4.1)[F(1,39)=73.066, p<.0001]$. Thus, subjective ratings on auditorily presented words varied as a function of both the sensory channel dimension and the performance dimension.

\section{Visually Presented Words}

A significant main effect was obtained for frequency $[F(2,39)=1209.95, p<.0001$; mean rating for high $=$ 8.1 ; mean rating for medium $=3.6$; mean rating for low $=$ 1.7]. Again, subjective ratings varied as a function of frequency of the stimulus items.

A significant main effect was also obtained for question $[F(3,39)=16.92, p<.0001$; mean rating for read $=$
4.6 ; mean rating for write $=4.2$; mean rating for hear $=$ 4.7 ; mean rating for say $=4.3$ ]. Planned contrasts were performed to determine whether ratings for visually presented words differed as a function of sensory channel and performance dimensions. Perception ratings were significantly higher than production ratings $[F(1,39)=$ $48.25, p<.0001]$. There was no effect of sensory channel for the visually presented words.

\section{Comparing Visually and \\ Auditorily Presented Words}

In order to compare ratings for visual and auditory presentations, an overall $4 \times 2 \times 3$ [question (read, write, hear, say) $\times$ modality (visual, auditory) $\times$ frequency (high, medium, low)] ANOVA was performed. Question and frequency were within-participants factors, and modality was a between-participants factor.

Main effects of question $[F(3,78)=44.30, p<.0001]$ and frequency $[F(2,78)=2173.56, p<.0001]$ were obtained. There was no main effect of modality $(F<1.0$; mean rating for visual $=4.5$; mean rating for auditory $=$ 4.5). However, modality was implicated in two signifi-
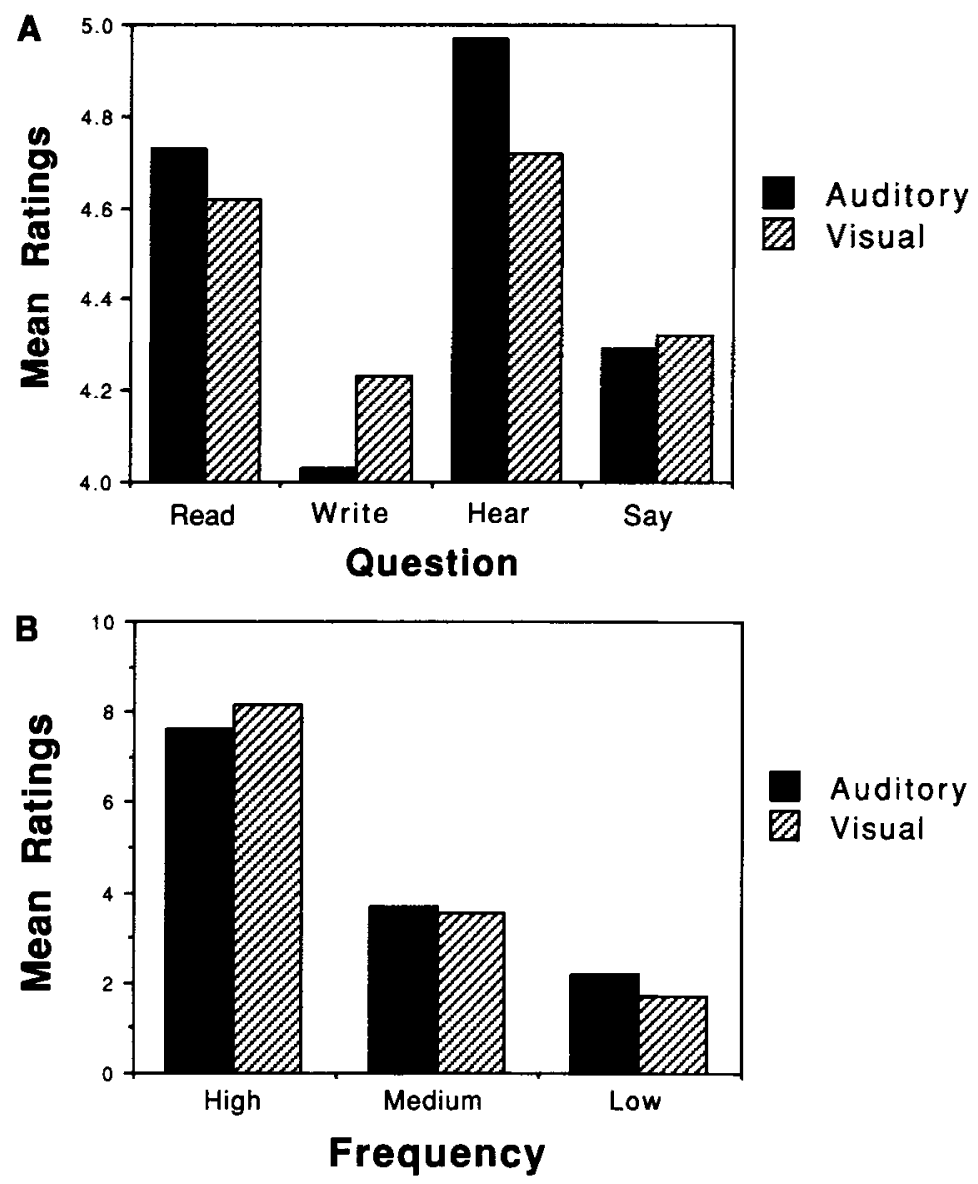

Figure 1. (A) Interaction between mode and question for the visual and auditory ratings. (B) Interaction between mode and frequency for the visual and auditory ratings. 
cant two-way interactions: (1) question and modality $[F(3,78)=3.78, p<.02]$ and $(2)$ frequency and modality $[F(2,1)=14.962, p=.0001]$.

These interactions are displayed in Figure 1. Figure 1A shows the interaction between modality and question. This interaction indicates that the read and hear questions resulted in higher ratings for auditorily presented words than for visually presented words, whereas the write and say questions resulted in higher ratings for visually presented words than for auditorily presented words. Figure 1B shows the interaction between modality and frequency. This interaction indicates that ratings varied as a function of modality within individual frequency levels. In particular, high-frequency words received lower mean ratings in the auditory modality than in the visual modality, whereas low-frequency words received lower mean ratings in the visual modality than in the auditory modality. These interactions provide support for the hypothesis that subjective frequency ratings are modality dependent. Moreover, these interactions argue against the application of general biases or strategies as a function of rating condition. Biases based on question would not be expected to vary with modality of presentation (or vice versa), nor would general strategies based on modality of presentation be expected to vary with frequency.

\section{Comparing Ratings Within and Across Modalities}

We correlated all ratings within and across modalities. Lower correlations between ratings processed in different modalities (e.g., visual read with auditory read, etc.) compared with correlations within the same modality (e.g., auditory read with auditory write, etc.) would provide evidence for modality dependence of subjective frequency ratings. This result would suggest that ratings for visually presented words and ratings for auditorily presented words tap different sources of information. Conversely, correlations of equal magnitude between ratings processed in the same modality and ratings processed in different modalities would indicate modality independence of subjective frequency ratings.

A schematic correlation matrix for read, write, hear, and say ratings in both visual and auditory modalities is shown in Figure 2. The portion of Figure 2 marked VIS/ VIS represents the correlation coefficients between individual questions in the visual modality. The portion of Figure 2 marked AUD/AUD represents the correlation coefficients between individual questions in the auditory modality. The portion marked VIS/AUD represents the correlation coefficients between individual questions across the visual and auditory modalities.

Correlations among individual questions within and across modalities are shown in Figure 3, in which each portion of the schematic correlation matrix in Figure 2 is represented by a bar. The upper panel of Figure 3 shows the mean correlation coefficients between individual questions within each modality (visual/visual and auditory/auditory) and across modalities (visual/auditory) for all frequencies. An insert on each bar shows the percentage of significant correlations $(p<.05)$ between individual questions in that modality. The same mean co-

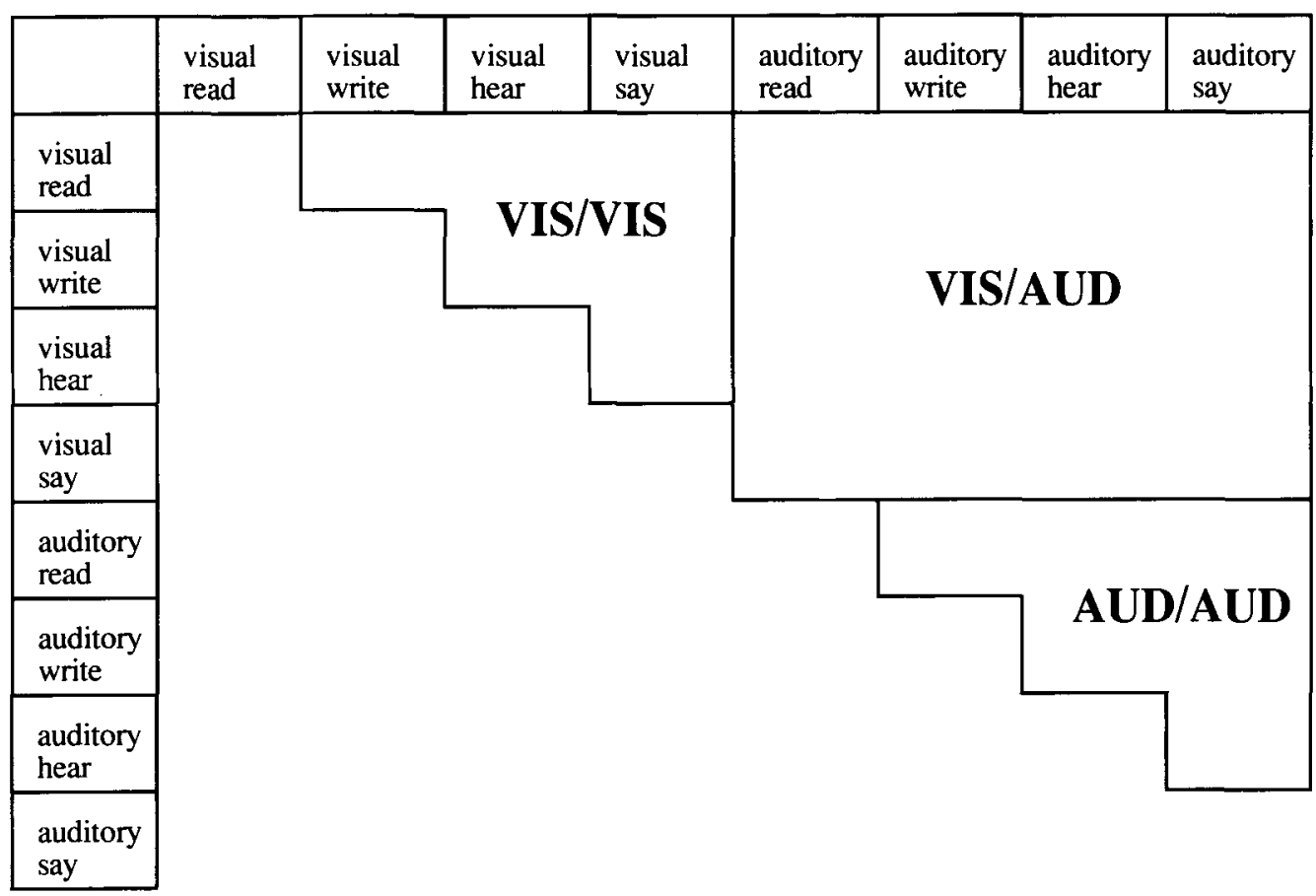

Figure 2. Schematic matrix for visual/visual (VIS/VIS), auditory/auditory (AUD/AUD), and visualauditory (VIS/AUD) correlations plotted in Figure 3. 

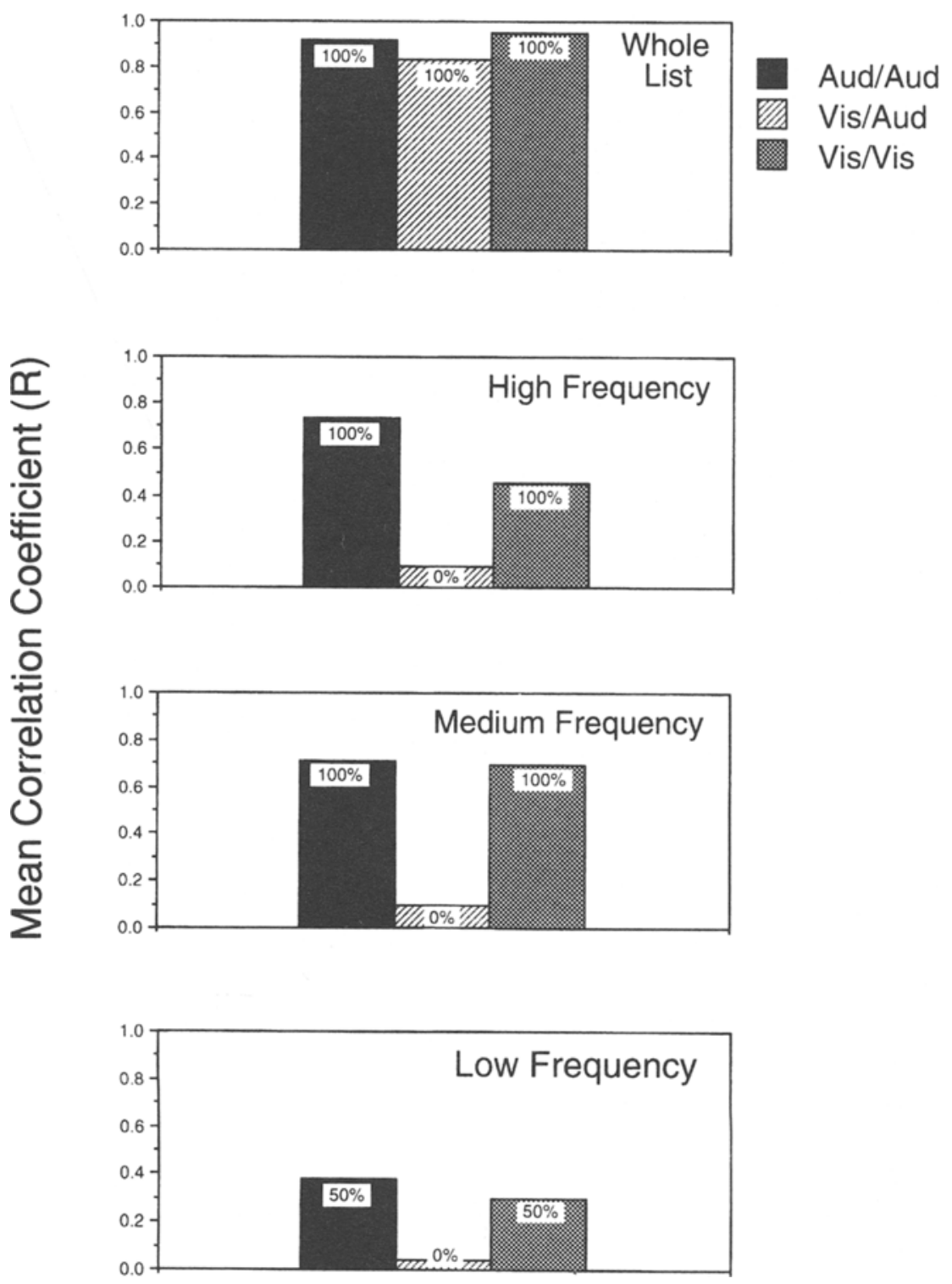

Figure 3. Mean correlation coefiicients for individual questions within and between modalities for the whole list and for the high-, medium-, and low-frequency sublists.

efficients for each frequency level (high, medium, and low) are also shown in Figure 3.

For the whole list, correlation coefficients between ratings in the same modality were very high. Although correlation coefficients between ratings across modalities were lower than within-modality correlation coefficients, they were still quite high. Viewed alone, this result suggests that subjective ratings of frequency are, to a large degree, modality independent. However, the figures for the three individual frequency levels reveal more dramatic differences in correlation coefficients among questions as a function of modality. (Note that the reduction in the magnitudes of the correlations for the sublists arises because of the restriction of the frequency range; see Cohen \& Cohen, 1983. Our intent is not to compare correlations for the sublists with those for the overall list but to compare correlations within and among the restricted frequency ranges themselves.) Within-modality correlation coefficients were much higher than betweenmodality correlation coefficients. For each frequency range, between-modality correlations were very low and were never significant. To summarize, correlation coeffi- 
cients between individual questions were high only within modality but significant correlation coefficients were nonexistent between modalities for the sublists.

It is important to note that the differences in the withinand between-modality correlations did not result from within- versus between-participants analyses. The correlations were based on the items, and no item was presented more than once to a given participant, regardless of modality. Thus, for each item, mean ratings for a condition (read, write, hear, say) are based on different groups of participants. For example, one group of participants rated the word south in the read condition, another group rated it in the write condition, and so on. The mean ratings from these different groups were then compared in order to determine the relationships between the read, write, hear, and say judgments. This procedure was employed for both auditorily and visually presented items. Therefore, all correlations were between participants, regardless of whether the correlations were computed within (VIS/VIS, AUD/AUD) or across (VIS/AUD) modalities.

Modality dependence is also suggested by an additional trend in the present data: The number and magnitude of significant correlations declined from high- to low-frequency words. Thus, agreement among read, write, hear, and say ratings was lowest for low-frequency words. This result suggests that specificity of access representations is more evident for low-frequency words than for high-frequency words.

\section{Discussion}

The participants gave differing assessments of word frequency based on the questions posed. Questions asking the participants how often they read or heard a word consistently resulted in higher ratings than did questions asking about the frequency of writing or saying. For both visually and auditorily presented words, the participants intuitions were that they are more likely to perceive words than to produce them. The participants' ratings reflect the intuitive notion that people typically encounter a higher volume of words in reading and listening than they do in speaking or writing.

In addition, the participants gave differing assessments of word frequency based on differences in modality of presentation of the target words. Questions asking the participants how often they read or heard a word resulted in consistently higher ratings when stimulus words were presented in the auditory modality than in the visual modality, whereas questions asking about writing and saying resulted in consistently higher ratings in the visual modality than in the auditory modality.

For auditorily presented words, ear ratings were higher than eye ratings. That is, the participants judged spoken words to be more frequent when modality of presentation and sensory channel of question matched. One possible source of this effect may stem from the modality specificity of the access representations. For example, stimulus words in the auditory modality may raise the activation levels of form-based representations of spoken words, which are then interrogated when participants are asked how frequently they hear or say a particular word. Thus, questions within the same modality as stimulus presentation may reflect the momentary increase in activation of the specific form-based representation.

This explanation should also presumably hold for visually presented words. However, we observed no such effect of modality of presentation on frequency ratings for visual words: Ear ratings were equivalent to eye ratings, suggesting that the participants may-on at least some trials-have been basing their judgments on phonologically recoded representations, thus masking effects of modality matching for the visual stimuli. Although speculative, this possibility is supported by evidence from Experiment 2 (see below). Whatever the precise mechanism underlying the interaction of question and modality, our results suggest that frequency judgments depend simultaneously (and in perhaps complex ways) on the nature of both the stimulus and the interrogative.

The pattern of correlation coefficients among individual questions within and across modalities is also evidence for modality dependence of subjective frequency ratings. For all frequencies combined, correlation coefficients among individual questions within both modalities were high and always significant. Correlation coefficients among individual questions across modalities were also high and always significant. However, the correlation coefficients among individual questions at each of the three frequency levels showed quite a different pattern. The correlation coefficients among individual questions within either modality were high and always significant for high- and medium-frequency words. For low-frequency words, the correlation coefficients within either modality were still fairly high and significant about half of the time. The correlation coefficients among individual questions across modalities, however, were always near zero and never significant.

In short, the participants made subjective word frequency judgments based on rather specific use criteria that varied as a function of sensory and performance dimensions as well as the modality of the target words. The present set of subjective ratings points to specificity of frequency indices in memory (see, however, Brown \& Watson, 1987). ${ }^{2}$ Four additional experiments were conducted in order to determine if these differences in ratings have implications for processing times. In order to address this issue, we presented the same visual and auditory words used in Experiments 1 in four word recognition paradigms in Experiment 2: (1) auditory lexical decision, (2) visual lexical decision, (3) auditory repetition, and (4) visual naming. These four tasks were chosen because they vary on modality of presentation (auditory 
vs. visual) and performance (each has a perception component; the repetition and naming experiments also have a production component). We then performed correlation and regression analyses to determine the degree of relationship between the subjective ratings and reaction times (RTs).

\section{EXPERIMENT 2 Processing}

Four versions of Experiment 2 were performed: auditory lexical decision, visual lexical decision, auditory repetition, and visual naming.

\section{Method}

\section{Participants}

Eighty undergraduates at the State University of New York at Buffalo participated in partial fulfillment of requirements for an introductory psychology course. Twenty participants served in each version of this experiment. All participants were native speakers of English, were right-handed, and reported no history of speech, hearing, or visual disorders.

\begin{abstract}
Materials
The stimuli were the same as those in Experiments $1 \mathrm{~A}$ and $1 \mathrm{~B}$. In addition, 252 nonwords were created for the auditory lexical decision experiment by changing one or two phonetic segments in a real word. Nonwords were created for visual lexical decision by varying one or two letters. The nonwords were all phonotactically legal in English. The spoken nonwords were produced by the same talker that produced the word stimuli. There were equal numbers of one-, two-, and three-syllable nonwords.
\end{abstract}

\section{Procedure}

Auditory lexical decision. The participants were seated in individual booths equipped with headphones and response boxes. Stimuli were output, and responses were collected by a PDP 11/34 computer. Order of presentation was randomized across all three word lists. A light on top of the response box flashed to signal the beginning of a new trial. On each trial, the participants responded as quickly and as accurately as possible whether a given auditorily presented stimulus was a word or a nonword. The participants responded "word" by pressing a button labeled "W" on the far right of a response box. They responded "nonword" by pressing a button labeled "N" on the far left of the response box. Responses were timed from the onset of the stimulus to the buttonpress response.

Visual lexical decision. The procedure was the same as that in auditory lexical decision except that the stimuli were presented visually on a computer display.

Auditory repetition. The participants were tested individually. On each trial, a word from the combined list was played over headphones. The participant's task was to repeat the word as quickly and as accurately as possible. Every participant repeated every word on the list. The participants wore headphones with a boom microphone attached. The boom microphone triggered a voice key that was interfaced to a PDP-11/34. The voice key was triggered at the onset of the participant's vocal response. Responses were timed from the onset of the stimulus until the onset of the participant's vocal response. The participants were instructed to avoid unrelated vocalization and any other unnecessary noise.

Visual naming. The procedure was the same as that in auditory repetition except that the stimuli were presented visually on a computer display.

\section{Results}

For each of the four processing paradigms, correlation and regression analyses were performed between mean ratings (independent variable) and RTs (dependent variable). Mean RTs were computed for each word in the high-, medium-, and low-frequency lists. ${ }^{3}$ The eight rating types [question (read, write, hear, say) $\times$ modality (visual, auditory) were correlated with and regressed against mean RTs for each list. Only correct responses were included in all analyses. Any repetition or naming trial that included an extraneous noise, such as a cough, or a hesitation was deleted from the analyses. For all versions of this experiment, latencies that exceeded \pm 2.5 standard deviations from the mean were excluded, and the means were recomputed. Removal of outliers resulted in nominally higher coefficients between each rating type and each latency type but did not alter the pattern of results in any version of Experiment 2. The latencies and errors for all versions of this experiment appear in Table 2. All latencies and error rates were within expected ranges for high-, medium-, and low-frequency words composed of one, two, and three syllables.

\section{Auditory Lexical Decision}

Correlation coefficients for each of the eight rating types and RTs for high-, medium-, and low-frequency words are shown in Figure 4. Regression coefficients are shown in Table 3. The four visual and auditory ratings are plotted on the $x$-axis, and the correlation coefficient $R$ is plotted on the $y$-axis. In both Figure 4 and Table 3, an asterisk indicates significance at the .05 level. For the high- and medium-frequency words, all and only the coefficients for auditory ratings were significant. For visual ratings of low-frequency words, all coefficients were significant. Finally, for the auditory ratings of the lowfrequency words, only coefficients for perception (read and hear) ratings were significant. ${ }^{4}$

\section{Visual Lexical Decision}

Correlation and regression coefficients for each of the eight rating types and RTs are shown in Figure 5 and Table 3. For the high-frequency words, only one coefficient was significant (auditory read). For the mediumfrequency words, all and only the coefficients for auditory ratings were significant. None of the coefficients was significant for the low-frequency words.

Table 2

Mean Latencies and Percent Errors for Experiment 2

\begin{tabular}{lrrrrr} 
& \multicolumn{2}{c}{ Latency $(\mathrm{msec})$} & & \multicolumn{2}{c}{ Error (\%) } \\
\cline { 5 - 6 } \cline { 5 - 6 } & $M$ & $S D$ & & \multicolumn{1}{c}{$S D$} \\
\hline Visual & & & & \\
$\quad$ Lexical Decision & 823 & 158 & 36.7 & 30.9 \\
Naming & 666 & 105 & 2.1 & 4.7 \\
Auditory & & & & \\
$\quad$ Lexical Decision & 1,029 & 168 & 30.6 & 29.5 \\
$\quad$ Repetition & 872 & 97 & 1.7 & 2.7 \\
\hline
\end{tabular}




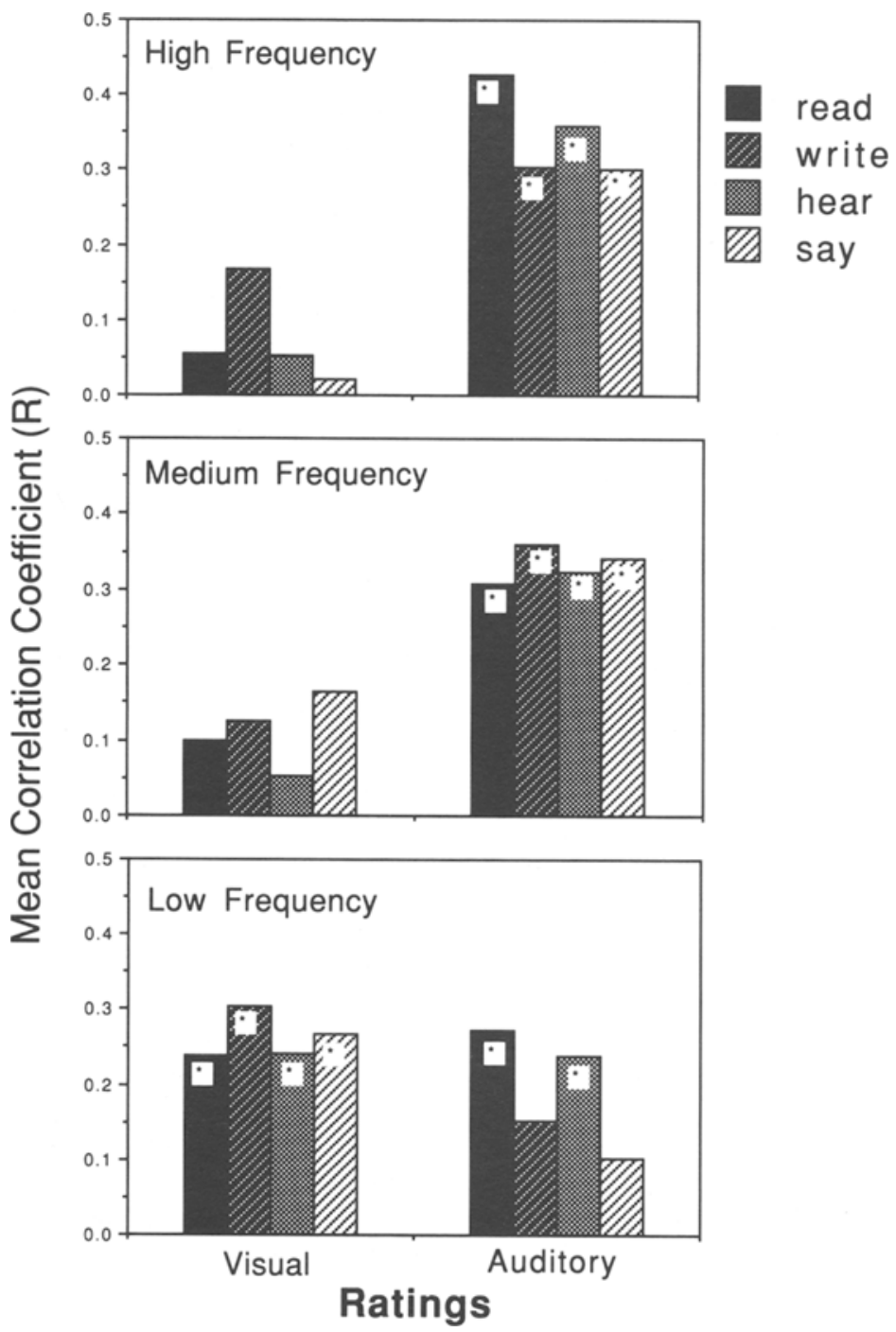

Figure 4. Mean correlation coefficients for auditory lexical decision for high-, medium-, and low-frequency words. An asterisk ( $\left.{ }^{*}\right)$ indicates significance at the .05 level.

\section{Auditory Repetition}

Correlation and regression coefficients are shown in Figure 6 and Table 3. Only two coefficients were significant (auditory read for high-frequency words, and auditory hear for low-frequency words).

\section{Visual Naming}

Correlation and regression coefficients are shown in Figure 7 and Table 3 . None of the coefficients was significant for high-frequency words. For medium-frequency words, only the coefficient for visual say was significant. For the visual ratings of low-frequency words, all coefficients were significant except for write. For the auditory ratings of low-frequency words, none of the coefficients was significant. ${ }^{5}$

\section{Discussion}

Four processing experiments were conducted in order to examine patterns of significant correlation and regression coefficients in word recognition experiments using visually and auditorily presented words. Although no consistent pattern of significant coefficients between individual questions and latencies occurred, a number of general patterns emerged. First, significant coefficients occurred more often for the visual and auditory lexical decision tasks (number of significant coefficients [hereafter, $n]=19$ ) than for the visual naming and auditory repetition tasks $(n=6)$. This result is consistent with previous findings in the literature that have demonstrated stronger frequency effects in lexical decision than in naming or repetition (Balota \& Chumbley, 1985; Luce, 
Table 3

Regression Coefficients for Experiment 2 for Ratings and Reaction Times

\begin{tabular}{|c|c|c|c|c|}
\hline & Read & Write & Hear & Say \\
\hline \multicolumn{5}{|c|}{ Auditory Lexical Decision } \\
\hline \multicolumn{5}{|c|}{ High-Frequency Words } \\
\hline Visual & 5.325 & -17.147 & 5.027 & -1.56 \\
\hline Auditory & $-28.864^{*}$ & $-18.153^{*}$ & $-23.905^{*}$ & $-16.015^{*}$ \\
\hline \multicolumn{5}{|c|}{ Medium-Frequency Words } \\
\hline Visual & -7.671 & -9.391 & -3.627 & -13.450 \\
\hline Auditory & $-26.009^{*}$ & $-35.048^{*}$ & $-24.020^{*}$ & $-27.373^{*}$ \\
\hline \multicolumn{5}{|c|}{ Low-Frequency Words } \\
\hline Visual & $-69.052^{*}$ & $-117.213^{*}$ & $-70.104^{*}$ & $-74.576^{*}$ \\
\hline Auditory & $-35.110^{*}$ & -23.514 & $-28.060^{*}$ & 18.468 \\
\hline \multicolumn{5}{|c|}{ Visual Lexical Decision } \\
\hline \multicolumn{5}{|c|}{ High-Frequency Words } \\
\hline Visual & 10.406 & -8.357 & -2.155 & 2.397 \\
\hline Auditory & -13.470 & -2.767 & -8.023 & -6.256 \\
\hline \multicolumn{5}{|c|}{ Medium-Frequency Words } \\
\hline Visual & 0.943 & 3.721 & 5.641 & -4.568 \\
\hline Auditory & $-29.331^{*}$ & $-34.631^{*}$ & $-25.100^{*}$ & $-29.116^{*}$ \\
\hline \multicolumn{5}{|c|}{ Low-Frequency Words } \\
\hline Visual & 1.402 & -17.546 & -53.077 & -19.797 \\
\hline Auditory & 3.017 & -10.398 & -.930 & -30.979 \\
\hline \multicolumn{5}{|c|}{ Auditory Repetition } \\
\hline \multicolumn{5}{|c|}{ High-Frequency Words } \\
\hline Visual & 0.035 & -13.506 & -0.173 & -4.247 \\
\hline Auditory & $-15.811^{*}$ & -7.306 & -10.125 & -9.661 \\
\hline \multicolumn{5}{|c|}{ Medium-Frequency Words } \\
\hline Visual & 6.160 & 3.906 & 1.413 & -1.538 \\
\hline Auditory & -3.812 & -8.112 & -6.216 & -2.280 \\
\hline \multicolumn{5}{|c|}{ Low-Frequency Words } \\
\hline Visual & -38.400 & -36.565 & -13.555 & -37.125 \\
\hline Auditory & $-17.14 !$ & -7.572 & $-24.107^{\star}$ & -13.278 \\
\hline \multicolumn{5}{|c|}{ Visual Naming } \\
\hline \multicolumn{5}{|c|}{ High-Frequency Words } \\
\hline Visual & 2.080 & -3.610 & -2.524 & -2.093 \\
\hline Auditory & 3.684 & 2.300 & 4.829 & 1.835 \\
\hline \multicolumn{5}{|c|}{ Medium-Frequency Words } \\
\hline Visual & -7.127 & -1.976 & -6.409 & -12.718 \\
\hline Auditory & -4.476 & -10.693 & -2.973 & -2.066 \\
\hline \multicolumn{5}{|c|}{ Low-Frequency Words } \\
\hline Visual & $-58.580^{*}$ & -34.611 & $-86.653^{*}$ & $-56.308^{*}$ \\
\hline Auditory & -4.570 & -18.552 & -14.809 & -26.620 \\
\hline
\end{tabular}

Note-"Read," "Write," "Hear," and "Say" indicate type of rating. "Visual" and "Auditory" indicate modality in which words were presented for subjective familiarity ratings. * ${ }^{*}$ Significant at $p<.05$.

1986). Second, significant coefficients occurred more often in the auditory tasks (lexical decision and repetition; $n=16$ ) than in the visual tasks (lexical decision and naming; $n=9$ ). Third, significant coefficients occurred more often for ratings made on auditorily presented stimuli $(n=17)$ than for ratings made on visually presented stimuli $(n=8)$.

Three more specific tendencies in the data were consistent with the hypothesis that access representations may have separate frequency indices in memory. In particular, for the visual naming experiment, significant coefficients were obtained only for visual ratings. Likewise, in the auditory repetitions experiment, significant coefficients were obtained only for auditory ratings. Finally, in the auditory lexical decision experiment, primarily au- ditory ratings resulted in significant coefficients (with a notable exception, see below). These three trends in the data support the hypothesis that frequency indices for access representations may have modality-specific qualities.

However, there are two trends in the data that appear, at first glance, to be inconsistent with the modalityspecificity hypothesis. In the auditory lexical decision experiment, significant coefficients for all visual ratings for low-frequency words were significant. Also, in visual lexical decision, only auditory ratings significantly predicted RTs.

Finally, we failed to see strong systematic trends in the coefficients as a function of word frequency or the specific ratings (read, write, hear, say). There was a slight trend for the number of significant coefficients to increase from high-frequency words to low-frequency words (high, $n=6$; medium, $n=9$; low, $n=10$ ). In addition, specific ratings for read, write, hear, and say failed to result in any clear-cut relationships with processing times, although there was a slight trend for perception ratings (read and hear; $n=15$ ) to result in more significant correlations than performance ratings (write and say; $n=10$ ).

\section{GENERAL DISCUSSION}

We examined subjective ratings of frequency of word usage for visually and auditorily presented words and the relationship of the subjective ratings to processing times in word recognition experiments. The results of Experiments 1 and 2 suggest that access representations in visual and spoken word recognition may have modalityspecific frequency indices in memory.

Forster (1976) offers a distinction between lexical entry and access representation. He defines lexical entry as a modality-independent core representation of a word's abstract syntactic, semantic, and phonetic properties. Forster defines access representation as modality-specific form representation that provides access to the core representations. The present study investigated the properties of the access representation.

The results of these experiments support the notion of modality-dependent frequency indices. The participants made different subjective judgments of frequency for words processed in the visual and auditory modalities. The access representations of visual and spoken words thus appear to incorporate differences in frequency of processing in the two modalities. The lack of significant correlations between subjective ratings of words processed in the visual and auditory modalities, relative to correlations for ratings processed in a single modality, suggests modality dependence of subjective frequency ratings. In addition, the mean perception ratings were consistently higher than the mean production ratings. ${ }^{6}$ We interpret this result as indicating that people generally perceive more words than they produce. Finally, the mean ear ratings were higher than the mean eye ratings for auditorily presented words. At some level, representations preserve frequency information specific to the 


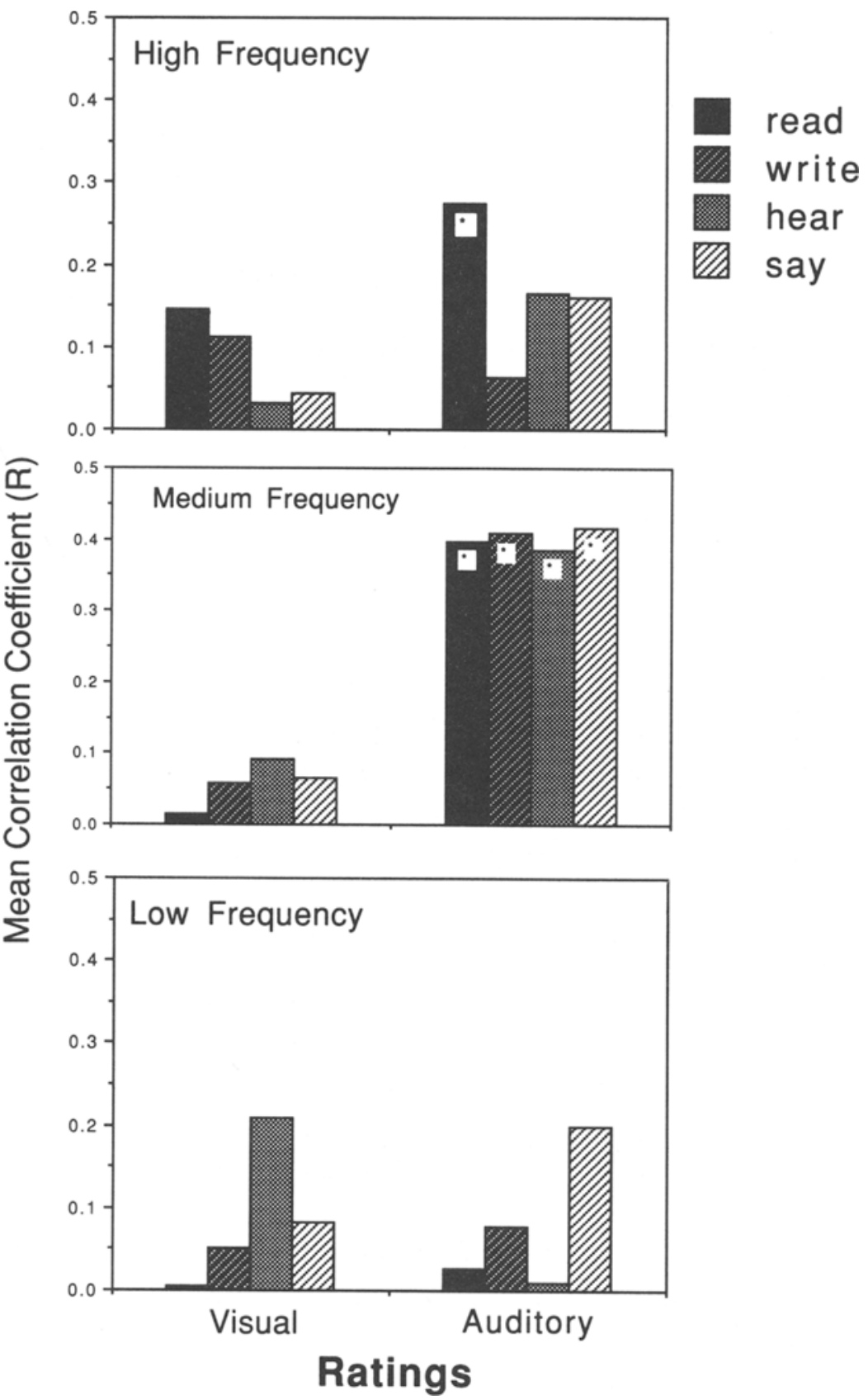

Figure 5. Mean correlation coefficients for visual lexical decision for high-, medium-, and low-frequency words. An asterisk $\left(^{*}\right)$ indicates significance at the .05 level.

dimensions of modality, perception, and performance. Form-based, modality-dependent access representations apparently possess their own frequency indices, which may be interrogated when participants are asked to rate subjective frequencies.

Subjective ratings may therefore inform us about modality dependence of lexical representations. Pisoni and Garber's (1990) conclusion that lexical representations are most likely modality independent is based on a high correlation between subjective ratings for words of varying frequency rated in the visual and auditory modalities. We found a similar high relationship for our combined list; however, our examination of each frequency level revealed lower relationships between words rated in the visual and auditory input modalities.

The finding that ratings and processing times tended to result in significant coefficients within a modality provides evidence for the hypothesis of separate frequency indices 


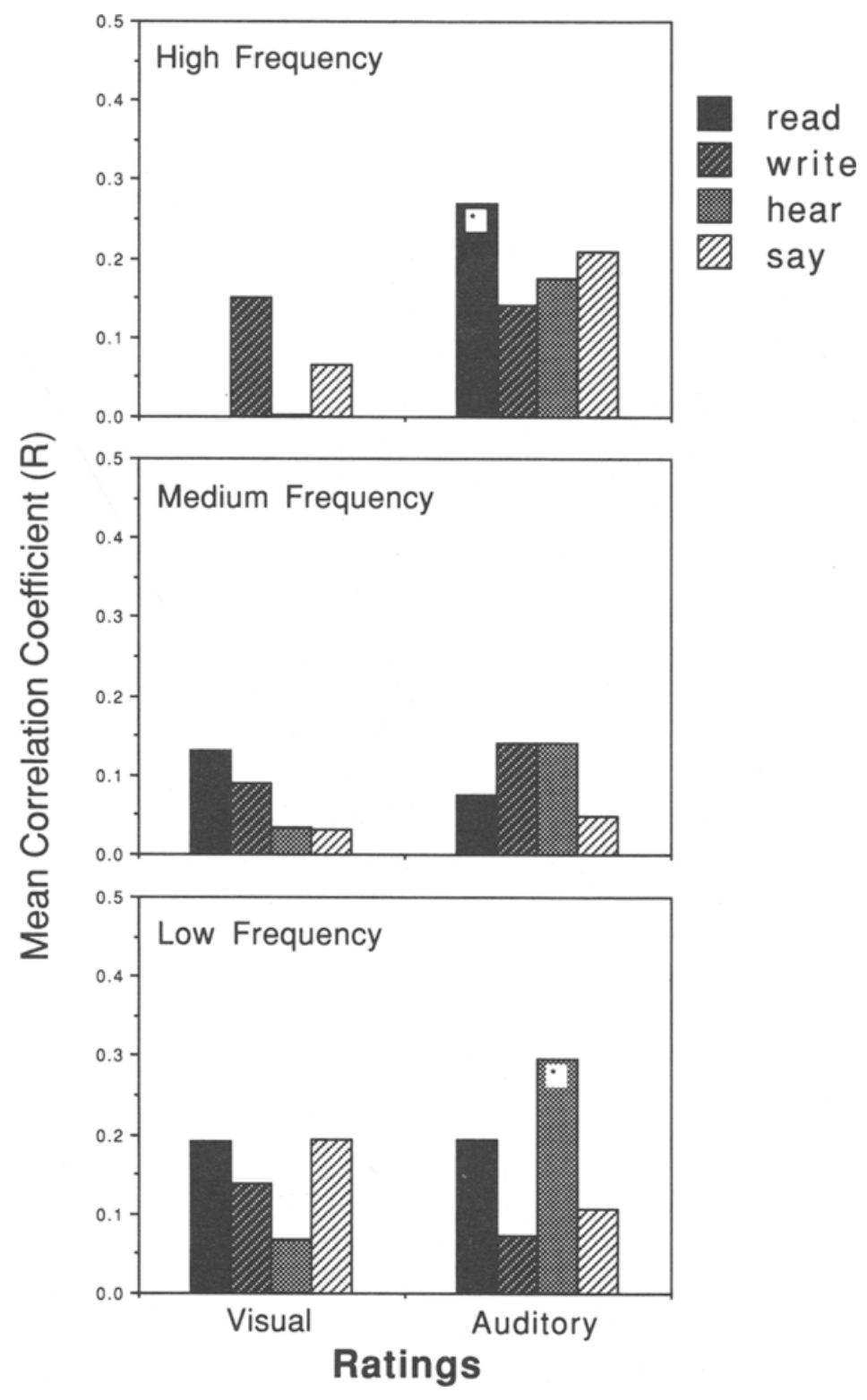

Figure 6. Mean correlation coefficients for auditory repetition for high-, medium-, and low-frequency words. An asterisk ( ${ }^{\star}$ ) indicates significance at the .05 level.

for modality-specific access representations. However, there were inconsistencies in the pattern of results. In particular, the finding that auditory ratings predicted visual lexical decision times appears, at first, problematic for the specificity account. However, if we assume that participants in our visual lexical decision task were generating phonologically recoded access representations prior to their responses, we would expect auditory, rather than visual, ratings to predict visual lexical decision processing times.

Our visual lexical decision experiment meets the standard criteria for inducing phonological recoding: (1) difficult word-nonword discrimination caused by the "word- like" nature of the nonword stimuli, and (2) an abundance of word stimuli that are "difficult" to process, primarily because of their multisyllabicity and low frequency. Both of these factors encourage phonological recoding in the lexical decision experiment (see McCusker, Hillinger, \& Bias, 1981). The absence of wordnonword discrimination in the visual naming task probably did not induce phonological recoding of the access representation itself, resulting in no significant correlations with auditory ratings for this task. (We assume that the articulatory response in the naming task is generated on the basis of a postaccess phonetic representation that 


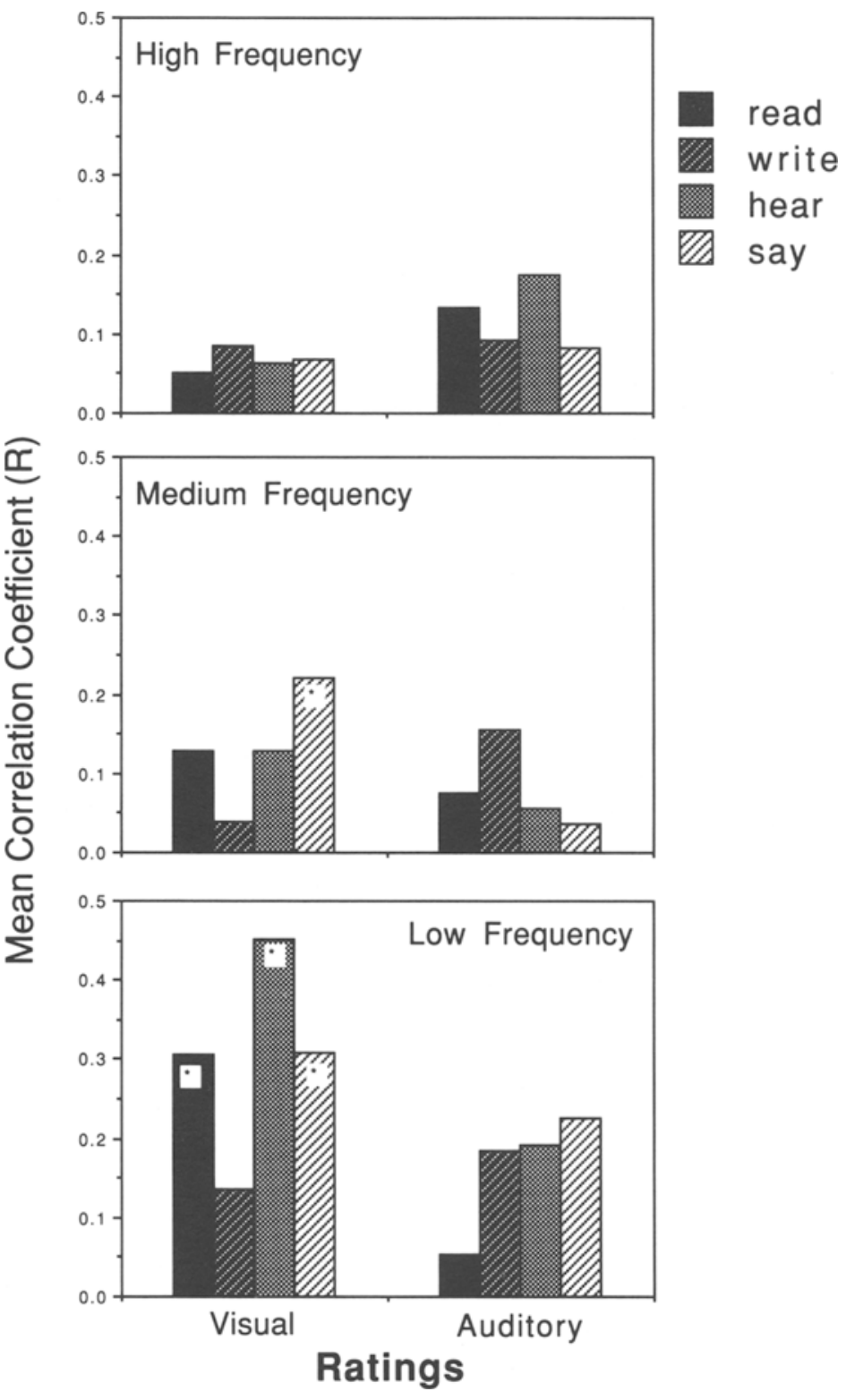

Figure 7. Mean correlation coefficients for visual naming for high-, mediumand low-frequency words. An asterisk $\left(^{*}\right)$ indicates significance at the .05 level.

does not affect access time; see Besner \& Johnston, 1989, and note 5.)

The failure to observe significant coefficients for the low-frequency words in the visual lexical decision experiment is also probably due to the use of phonologically recoded representations, which overall resulted in few significant coefficients for RTs for low-frequency words in any of the experiments. Indeed, in the one experiment in which auditory representations resulted in the strongest coefficients overall (namely, auditory lexical decision), the weakest predictors of RTs were the auditory ratings for low-frequency words. (See below for further discussion of the use of auditory-based access representations for low-frequency words.)
The specificity hypothesis is also called into question by the finding that all four visual ratings resulted in significant coefficients for low-frequency words in the auditory lexical decision experiment. This result may have arisen because we have more experience with lowfrequency words in the visual modality (specifically, in print) than in the auditory modality. Indeed, we may be able to recognize many low-frequency words in print in the absence of stable phonetic representations in memory. Thus, we speculate that participants may be consulting orthographically based representations for low-frequency words in the auditory lexical decision experiment in the absence of well-defined phonetic codes. In essence, we suggest that participants may often be "orthographically 
recoding" the low-frequency spoken words to assist them in making lexical decisions to words with which they have had little or no experience in spoken discourse. This activation of orthographic representations may thus be producing the significant relationships that we observed between the visual ratings and auditory lexical decision times for low-frequency words.

A coherent account of these results is, at present, more speculative than definitive. Nonetheless, we propose that access representations have modality-specific frequency indices that arise from differential experience with words in different modalities. For the most part, auditory tasks will invoke auditory representations, and visual tasks will invoke visual representations. However, we furthermore propose that the modality-specific access representation with the higher frequency may dominate participants' responses under certain conditions, regardless of task modality. For example, it is obvious that we have more experience with low-frequency words in print than in spoken discourse (and our ratings for items presented in the visual modality bear this out). We may infer, therefore, that visual representations tend to dominate for low-frequency words, thus producing the pattern of relationships observed in the auditory lexical decision experiment. (We might expect the same pattern to hold in the repetition task, were it not for markedly attenuated frequency effects typically observed in this paradigm; see Balota \& Chumbley, 1985, and Luce, 1986.) In short, the patterns of significant coefficients for the processing time data are consistent overall with a modality-specific frequency account, although local inconsistencies in the data point to the need for future research on the specifics of our proposed explanation.

One avenue for future research would be to investigate the relationships among processing times, the subjective ratings in the present experiment, and other objective and subjective measures of familiarity and frequency (including age of acquisition norms; Morrison \& Ellis, 1995). As an initial step toward this goal, we performed overall correlations between the Nusbaum et al. (1984) subjective familiarity ratings, the Kučera and Francis (1967) objective counts, and the processing times obtained in all of our experiments. Overall, our subjective frequency ratings and the Nusbaum et al. subjective familiarity ratings resulted in equivalent correlation coefficients. (This result is hardly surprising given the high correlation between the subjective familiarity and frequency ratings; see note 2.) However, our subjective ratings resulted in consistently higher correlations than the objective counts based on Kučera and Francis. In short, our ratings appear overall to be quite similar to the Nusbaum et al. subjective familiarity ratings but somewhat stronger predictors of processing times than the objective counts.

In summary, the present data are consistent with the spirit of both Morton's (1969) and Forster's (1976) models of word recognition. Later versions of Morton's logogen model incorporate separate logogen systems for written and spoken words, explicitly acknowledging modality- dependent frequency information. The present data are also generally consistent with Forster's autonomous search model, although our findings suggest that processing may not be limited to a single, modality-specific peripheral access bin during recognition.

Our results are consistent with a growing trend in modeling work on word recognition (see, e.g., Goldinger, 1992) to consider the nature and specificity of peripheral access representations, a theme introduced by the early models of Forster and Morton (see Howard, 1995, for a further discussion on specificity and separate input and output phonological systems). We believe that any comprehensive model of word recognition must account for recent findings that such specific information as the voice of the talker (Mullennix, Pisoni, \& Martin, 1989; Pisoni, 1990) and allophonic information (Luce, Lyons, \& Myers, 1994) are encoded in lexical representations at some level in the processing system.

Evidence for the specificity of lexical representations is consistent with exemplar memory models, such as that proposed by Hintzman (1988), which may account for the distinctions made by the participants' frequency judgments in the present study. Each use episode may be encoded as a distinct entry at the access representation level and may explicitly encode features identifying that episode as having emanated from the visual or auditory modality, perception or production, eye or ear channel, and so on. This would not preclude a separate lexical representation that may be much more abstract. It would also not preclude such a lexical representation from having frequency information of its own. Nonetheless, the next generation of comprehensive lexical processing models may benefit from a synthesis of insights from the earliest models of word recognition and current work on exemplar-based representations in memory.

\section{REFERENCES}

Amano, S., Kondo, T., \& KaKehi, K. (1995). Modality dependency of familiarity ratings of Japanese words. Perception \& Psychophysics, 57, 598-603.

Balota, D. A., \& Chumbley, J. I. (1985). The locus of word-frequency effects in the pronunciation task: Lexical access and/or production? Journal of Memory \& Language, 24, 89-106.

BESNER, D., \& Johnston, J. C. (1989). Reading and the mental lexicon: On the uptake of visual information. In W. Marslen-Wilson (Ed.), Lexical representation and process (pp. 291-316). Cambridge, MA: MIT Press, Bradford Books.

Brown, G. D. A., \& Watson, F. L. (1987). First in, first out: Word learning age and spoken word frequency as predictors of word familiarity and word naming latency. Memory \& Cognition, 15, 208-216.

COHEN, J., \& COHEN, P. (1983). Applied multiple regression/correlation analysis for the behavioral sciences ( $2 \mathrm{nd}$ ed.). Hillsdale, $\mathrm{NJ}$ : Erlbaum.

Connine, C. M., Mullennix, J., Shernoff, E., \& Yelen, J. (1990). Word familiarity and frequency in visual and auditory word recognition. Journal of Experimental Psychology: Learning. Memory, \& Cognition, 16, 1084-1096.

DELL, G. S. (1990). Effects of frequency and vocabulary type on phonological speech errors. Language \& Cognitive Processes, 4, 313-349.

Forster, K. I. (1976). Accessing the mental lexicon. In R. J. Wales \& E. Walker (Eds.), New approaches to language mechanisms (pp. 257284). Amsterdam: North-Holland.

ForSTER, K. I., \& ChAMBERS, S. M. (1973). Lexical access and naming time. Journal of Verbal Learning \& Verbal Behavior, 12, 627-635. 
Garber, E. E., \& Pisoni, D. B. (1991). Lexical memory in visual and auditory modalities: A second report. In Research on speech perception (Progress Rep. No. 17, pp. 213-227). Bloomington: Indiana University, Department of Psychology, Speech Research Laboratory.

GeRNSBACHER, M. A. (1984). Resolving 20 years of inconsistent interactions between lexical familiarity and orthography, concreteness and polysemy. Journal of Experimental Psychology: General, 113, 256-281.

GOLDINGER, S. D. (1992). Words and voices: Implicit and explicit memory for spoken words. Dissertation Abstracts International, 53(6-B), 3189.

HinTZMAN, D. L. (1988). Judgments of frequency and recognition memory in a multiple trace memory model. Psychological Review, 95, 528-551.

Howard, D. (1995). Lexical anomia: Or the case of the missing lexical entries. Quarterly Journal of Experimental Psychology, 48A, 999-1023.

JESCHENIAK, J. D., \& LeVELT, W. J. M. (1994). Word frequency effects in speech production: Retrieval of syntactic information and of phonological form. Journal of Experimental Psychology: Learning, Memory, \& Cognition, 20, 824-843.

Kư̌ERA, H., \& FranCIS, W. N. (1967). Computational analysis of presentday American English. Providence, RI: Brown University Press.

LuCE, P. A. (1986). Neighborhoods of words in the mental lexicon. Unpublished doctoral dissertation, Indiana University, Bloomington.

LUCE, P. A., LyONS, E. A., \& MYERS, J. (1994). The abstractness and specificity of lexical representations in memory: Implications for models of spoken word recognition. In Proceedings of the International Conference on Spoken Language Processing. Yokohama, Japan: The Acoustical Society of Japan.

LuCE, P. A., Pisoni, D. B., \& Goldinger, S. (1990). Similarity neighborhoods of spoken words. In T. M. Altman (Ed.), Cognitive models of speech processing: Psycholinguistic and computational perspectives (pp. 122-147). Cambridge, MA: MIT Press.

McClelland, J. L., \& RumelhaRT, D. E. (1981). An interactive activation model of context effects in letter perception: I. An account of basic findings. Psychological Review, 88, 375-407.

MCCusker, L. X., Hillinger, M. L., \& Bias, R. G. (1981). Phonological recoding and reading. Psychological Review, 89, 217-245.

MorRISON, C. M., \& ELLIS, A. W. (1995). Roles of word frequency and age of acquisition in word naming and lexical decision. Journal of Experimental Psychology: Learning, Memory, \& Cognition, 21, 116-133.

Morton, J. (1969). Interaction of information in word recognition. Psychological Review, 76, 165-178.

MORTON, J. (1979). Facilitation in word recognition: Experiments causing change in the logogen model. In P. A. Kolers, M. E. Wrolstad, \& H. Bouma (Eds.), Processing of visible language (pp. 259-268). New York: Plenum.

MullenniX, J. W., Pisoni, D. B., \& Martin, C. S. (1989). Some effects of talker variability on spoken word recognition. Journal of the Acoustical Society of America, 85, 365-378.

Nusbaum, H. C., Pisoni, D. B., \& Davis, C. K. (1984). Sizing up the Hoosier mental lexicon: Measuring the familiarity of 20,000 words. In Research on speech perception (Progress Rep. No. 10). Bloomington: Indiana University, Speech Research Laboratory, Department of Psychology.

PisONI, D. B. (1990). Effects of talker variability on speech perception: Implications for current research and theory. In H. Fujisaki (Ed.), Proceedings of the 1990 International Conference on Spoken Language Processing (pp. 1399-1407). Kobe, Japan: The Acoustical Society of Japan.

Pisoni, D. B., \& Garber, E. E. (1990). Lexical memory in visual and auditory modalities: The case for a common mental lexicon. In $\mathrm{H}$. Fujisaki (Ed), Proceedings of the 1990 International Conference on Spoken Language Processing (pp. 401-404). Kobe, Japan: The Acoustical Society of Japan.

THORNDIKE, E. L., \& LORGE, I. (1944). The teacher's word book of 30,000 words. New York: Columbia University, Teachers College, Bureau of Publications.

Webster's Seventh Collegiate Dictionary (1967). Los Angeles: Library Reproduction Service.

\section{NOTES}

1. In all analyses, visual and auditory stimuli that may have been ambiguous were eliminated prior to data analysis. For visually presented items, we eliminated those items with two possible lexical stress patterns (e.g., recorD and REcord). For the spoken words, we presented the stimuli to an independent group of participants and asked them to write down what they heard. Those words that were incorrectly identified were eliminated. This resulted in the elimination of 25 stimuli from the original set of 252 stimuli. In no case were the results affected by the elimination of ambiguous items. The results for the trimmed stimulus set are reported.

2. Previous studies on the modality specificity of frequency indices in memory have used measures of subjective familiarity (Amano et al., 1995; Pisoni \& Garber, 1990). It is possible that subjective and objective measures of familiarity and frequency tap different aspects of participants' experience with spoken and written words. We correlated our measure of subjective frequency with subjective measures of familiarity (Nusbaum et al., 1984) and objective measures of frequency (Kučera \& Francis, 1967) previously employed in the literature. For both auditory and visual ratings, we obtained strong, significant correlations between the subjective familiarity ratings employed in Pisoni and Garber (1990; see Nusbaum et al., 1984) ( $r$ s $=.81$ and .90 for auditory and visual, respectively) and between the Kučera and Francis (1967) norms $(r s=.84$ and .88 for auditory and visual, respectively). Although there is some small proportion of unexplained variance in these correlations, we are confident that, for the most part, our ratings of subjective frequency measure essentially the same frequency information in memory indexed by subjective familiarity and objective frequency counts. Although the unexplained variance in the present correlations is most probably due to technical differences, such as variations in subject populations (both in location and in time), it may be of interest in future research to compare these measures directly in the same experimental context in order to determine whether different measures of frequency or familiarity tap different types of information in memory.

3. Because our intent is to compare ratings within the specific frequency ranges of high, medium, and low, we report regression coefficients $(\beta \mathrm{s})$ in addition to the correlation coefficients $(R \mathrm{~s})$. Regression coefficients are not subject to problems of range restriction encountered with correlations (see Cohen \& Cohen, 1983). Thus, comparisons among the frequency ranges are made more tractable by examining regression coefficients.

4. Because RTs in the auditory lexical decision experiment were measured from the onsets of the stimuli, it was necessary to establish that any observed relationships between subjective frequency and RT were not in fact due to relationships between subjective frequency and stimulus duration. We therefore correlated stimulus duration and subjective auditory frequency ratings for each frequency range. No significant correlation was obtained, and the average of the correlations was -.06 .

5. Naming and repetition latencies may reflect characteristics of the stimulus to be articulated, producing contaminated measures of lexical access time. In particular, the voice key may be differentially sensitive to the phonetic characteristics of the initial segment of the word to be articulated. One means of evaluating these potential confounds is to present a delayed cue for the naming or repetition response well after the stimulus word has been accessed (see Balota \& Chumbley, 1985). Any correlations remaining in the delayed naming or repetition condition can thus be attributed not to access - which should have occurred long before the cued response- but to stimulus characteristics. Likewise, the failure to obtain effects in the delayed condition that were observed in immediate naming or repetition can more safely be attributed to processes involved in access. Therefore, we ran two additional experiments: delayed auditory repetition and delayed visual naming with intervals between stimulus presentation and response cue of $1,200 \mathrm{msec}$. We computed correlations between the latencies for the delayed auditory repetition and delayed visual naming latencies in the present study with all ratings at all frequency ranges. No rating type at any frequency level correlated significantly with the delayed repetition or delayed naming latencies. We are therefore confident that the significant corre- 
lations we found in the immediate repetition and naming experiments were due to lexical processing and not stimulus characteristics.

6. At present, the precise locus of frequency effects in spoken word production is controversial (see Dell, 1990; Jescheniak \& Levelt, 1994). Our intent in this research was not to identify the loci of frequency effects in production but to attempt to ascertain whether ratings based on different types of questions correlate with processing times measured in the naming and repetition tasks. To the extent that we obtained significant correlations between ratings and naming and repetition, we believe our results implicate modality-specific frequency effects in word production. The precise locus of these correlations, however, will require further research (see, however, Jescheniak \& Levelt, 1994).

\section{APPENDIX}

Stimulus Words and Ratings for Each Question Condition (Read [R], Write [W], Hear [H], Say [S]) for Each Presentation Modalilty (Visual, Auditory)

\begin{tabular}{|c|c|c|c|c|c|c|c|c|}
\hline \multirow[b]{2}{*}{ Stimulus Words } & \multicolumn{4}{|c|}{ Visual } & \multicolumn{4}{|c|}{ Auditory } \\
\hline & $\mathbf{R}$ & W & $\mathbf{H}$ & $\mathbf{S}$ & $\mathbf{R}$ & W & $\mathrm{H}$ & $\mathrm{S}$ \\
\hline accolade & 2.80 & 1.50 & 2.50 & 2.10 & 1.89 & 1.80 & 1.50 & 1.50 \\
\hline acumen & 1.90 & 1.70 & 2.00 & 1.60 & 2.80 & 2.80 & 3.30 & 1.00 \\
\hline afternoon & 8.00 & 8.20 & 7.90 & 8.10 & 6.80 & 4.60 & 8.20 & 6.60 \\
\hline aggregate & 6.10 & 5.10 & 5.30 & 4.60 & 4.40 & 2.60 & 3.80 & 2.10 \\
\hline akin & 3.10 & 1.90 & 1.50 & 2.20 & 3.20 & 2.30 & 2.70 & 1.80 \\
\hline albumen & 1.70 & 2.10 & 1.70 & 1.20 & 2.10 & 1.40 & 2.90 & 1.40 \\
\hline amortize & 1.30 & 2.60 & 1.70 & 1.40 & 2.70 & 2.50 & 2.90 & 1.10 \\
\hline angst & 1.50 & 1.50 & 3.00 & 1.30 & 3.20 & 1.90 & 3.20 & 2.70 \\
\hline apogee & 1.30 & 2.20 & 3.00 & 1.30 & 1.30 & 1.60 & 1.30 & 2.10 \\
\hline appanage & 1.60 & 1.30 & 1.70 & 1.30 & 1.60 & 1.10 & 1.50 & 1.10 \\
\hline audience & 8.30 & 6.30 & 7.70 & 9.30 & 2.50 & 2.00 & 3.70 & 2.60 \\
\hline average & 7.70 & 9.22 & 8.10 & 8.40 & 6.30 & 6.30 & 7.50 & 6.90 \\
\hline ballistic & 3.40 & 4.00 & 4.20 & 3.00 & 3.40 & 4.10 & 6.30 & 5.30 \\
\hline ballyhoo & 1.60 & 1.50 & 2.20 & 1.10 & 1.50 & 1.50 & 3.00 & 2.11 \\
\hline beatnik & 2.60 & 1.80 & 1.70 & 1.20 & 2.50 & 1.90 & 4.40 & 3.90 \\
\hline beaujolais & 2.50 & 1.20 & 1.20 & 1.00 & 2.00 & 1.30 & 1.00 & 2.30 \\
\hline bivouac & 1.50 & 1.20 & 1.40 & 1.20 & 1.60 & 1.60 & 1.60 & 2.10 \\
\hline bolo & 1.60 & 1.50 & 1.70 & 1.30 & 2.00 & 2.00 & 2.30 & 2.30 \\
\hline book & 8.80 & 7.40 & 9.40 & 9.20 & 9.00 & 7.60 & 9.80 & 9.50 \\
\hline bout & 3.60 & 2.11 & 4.40 & 4.00 & 3.60 & 3.60 & 4.10 & 2.30 \\
\hline bracken & 3.30 & 1.70 & 1.70 & 1.60 & 2.30 & 2.20 & 2.60 & 2.10 \\
\hline bravado & 3.00 & 3.40 & 3.20 & 2.80 & 3.50 & 2.80 & 3.90 & 2.00 \\
\hline brethren & 3.20 & 1.90 & 2.40 & 1.30 & 5.20 & 3.30 & 4.60 & 3.00 \\
\hline bucolic & 1.20 & 1.10 & 1.00 & 1.10 & 2.60 & 1.70 & 1.10 & 2.10 \\
\hline call & 7.40 & 8.00 & 8.70 & 8.67 & 8.20 & 7.20 & 8.40 & 9.50 \\
\hline cantonment & 2.70 & 1.20 & 1.20 & 2.90 & 1.89 & 1.50 & 1.50 & 2.20 \\
\hline carabao & 1.80 & 1.00 & 2.10 & 1.33 & 1.60 & 2.30 & 1.67 & 2.10 \\
\hline care & 7.60 & 8.20 & 8.60 & 8.10 & 8.60 & 8.30 & 8.60 & 8.40 \\
\hline cathode & 4.00 & 3.00 & 3.80 & 3.40 & 2.00 & 1.40 & 2.40 & 1.70 \\
\hline celestial & 4.60 & 4.20 & 5.10 & 5.10 & 4.30 & 2.30 & 2.90 & 2.70 \\
\hline central & 8.30 & 7.20 & 8.30 & 6.44 & 8.70 & 6.30 & 8.20 & 9.10 \\
\hline century & 6.90 & 8.30 & 7.80 & 7.20 & 7.50 & 5.40 & 5.70 & 6.70 \\
\hline chevalier & 2.50 & 1.30 & 1.70 & 1.50 & 3.60 & 3.10 & 3.70 & 2.10 \\
\hline clad & 2.60 & 2.78 & 3.20 & 2.10 & 4.50 & 4.10 & 5.70 & 4.90 \\
\hline claimant & 3.00 & 1.60 & 4.10 & 1.60 & 3.80 & 4.20 & 3.80 & 2.30 \\
\hline clung & 5.70 & 3.60 & 3.00 & 4.10 & 3.90 & 3.10 & 5.30 & 4.00 \\
\hline coalesce & 2.10 & 2.80 & 3.10 & 2.40 & 2.30 & 1.20 & 1.50 & 2.50 \\
\hline common & 8.60 & 8.50 & 8.30 & 8.50 & 7.50 & 7.10 & 8.40 & 8.10 \\
\hline compiler & 2.60 & 4.40 & 4.30 & 1.80 & 4.60 & 4.10 & 6.10 & 3.70 \\
\hline concord & 4.00 & 2.20 & 5.90 & 3.60 & 2.20 & 1.10 & 2.20 & 1.30 \\
\hline contention & 4.50 & 4.40 & 4.10 & 4.40 & 4.00 & 2.70 & 3.20 & 2.70 \\
\hline cost & 8.90 & 8.40 & 9.00 & 8.90 & 8.40 & 8.40 & 8.40 & 8.50 \\
\hline creche & 2.30 & 1.50 & 1.30 & 1.50 & 1.20 & 1.30 & 3.50 & 3.00 \\
\hline credo & 2.30 & 1.30 & 2.70 & 1.60 & 3.30 & 2.70 & 4.70 & 5.40 \\
\hline cur & 1.80 & 1.30 & 1.50 & 1.40 & 1.40 & 2.20 & 3.40 & 1.70 \\
\hline cusp & 2.50 & 2.50 & 2.40 & 2.00 & 1.60 & 1.80 & 1.50 & 2.80 \\
\hline daily & 7.50 & 8.50 & 8.70 & 8.11 & 8.10 & 5.00 & 7.60 & 7.10 \\
\hline decision & 9.00 & 8.30 & 8.30 & 9.30 & 7.30 & 6.60 & 7.50 & 5.10 \\
\hline deep & 9.00 & 7.30 & 8.70 & 9.00 & 7.60 & 6.10 & 6.40 & 6.10 \\
\hline defense & 9.20 & 7.50 & 8.60 & 8.00 & 8.10 & 8.80 & 9.00 & 8.20 \\
\hline divest & 3.50 & 2.10 & 2.20 & 4.60 & 3.70 & 4.20 & 3.30 & 1.90 \\
\hline
\end{tabular}


APPENDIX (Continued)

\begin{tabular}{|c|c|c|c|c|c|c|c|c|}
\hline \multirow[b]{2}{*}{ Stimulus Words } & \multicolumn{4}{|c|}{ Visual } & \multicolumn{4}{|c|}{ Auditory } \\
\hline & $\mathbf{R}$ & W & $\mathrm{H}$ & $\mathbf{S}$ & $\mathbf{R}$ & W & $\mathrm{H}$ & $\mathbf{S}$ \\
\hline druid & 2.50 & 2.10 & 1.70 & 2.10 & 1.90 & 1.10 & 2.30 & 1.90 \\
\hline east & 9.30 & 7.60 & 7.30 & 9.60 & 8.60 & 8.40 & 8.30 & 7.80 \\
\hline eclat & 1.70 & 1.20 & 1.90 & 1.20 & 2.80 & 1.10 & 2.10 & 2.10 \\
\hline esplanade & 1.80 & 1.10 & 1.50 & 1.10 & 1.40 & 1.50 & 1.50 & 2.00 \\
\hline evidence & 8.20 & 7.50 & 8.00 & 7.10 & 7.30 & 8.50 & 8.30 & 7.60 \\
\hline example & 9.80 & 8.60 & 8.80 & 9.60 & 8.90 & 7.90 & 8.70 & 7.40 \\
\hline existence & 8.10 & 7.10 & 8.60 & 7.30 & 6.00 & 6.80 & 7.90 & 5.40 \\
\hline facade & 4.30 & 5.90 & 4.00 & 3.90 & 3.60 & 2.00 & 5.60 & 3.30 \\
\hline fall & 8.50 & 8.80 & 9.10 & 8.50 & 6.90 & 5.20 & 7.40 & 6.60 \\
\hline farm & 7.70 & 6.90 & 6.90 & 6.80 & 7.50 & 7.30 & 7.20 & 7.40 \\
\hline fecund & 1.20 & 1.20 & 1.40 & 1.40 & 3.10 & 1.78 & 1.70 & 2.30 \\
\hline fetid & 1.40 & 1.30 & 2.20 & 1.60 & 1.80 & 2.00 & 2.20 & 1.80 \\
\hline final & 9.11 & 7.90 & 9.50 & 8.60 & 8.30 & 8.30 & 8.70 & 8.40 \\
\hline finally & 8.60 & 8.80 & 8.30 & 9.50 & 8.20 & 8.90 & 8.80 & 8.10 \\
\hline firm & 7.50 & 7.50 & 8.20 & 7.40 & 7.60 & 6.70 & 7.50 & 7.10 \\
\hline flux & 4.60 & 3.30 & 4.80 & 4.60 & 3.50 & 2.80 & 3.20 & 4.10 \\
\hline fresco & 3.50 & 2.20 & 2.30 & 2.90 & 2.60 & 2.60 & 2.10 & 2.70 \\
\hline frontage & 3.20 & 1.70 & 3.50 & 4.20 & 2.30 & 3.20 & 2.40 & 3.00 \\
\hline gaiety & 3.10 & 1.50 & 2.50 & 1.80 & 4.90 & 3.90 & 6.10 & 3.10 \\
\hline gentian & 1.90 & 1.80 & 1.50 & 1.30 & 1.40 & 1.00 & 1.90 & 2.20 \\
\hline gradient & 6.20 & 4.10 & 4.50 & 4.70 & 4.40 & 3.50 & 4.30 & 4.20 \\
\hline grist & 1.50 & 1.90 & 1.60 & 2.10 & 2.22 & 2.60 & 2.30 & 3.30 \\
\hline groat & 3.10 & 1.90 & 1.90 & 2.10 & 1.40 & 1.20 & 2.80 & 2.20 \\
\hline gun & 7.50 & 8.60 & 8.30 & 6.90 & 8.70 & 7.30 & 8.60 & 9.10 \\
\hline hap & 1.60 & 1.40 & 3.30 & 2.40 & 1.80 & 1.50 & 1.60 & 2.90 \\
\hline hit & 7.70 & 8.20 & 9.30 & 8.30 & 7.40 & 6.20 & 8.20 & 8.10 \\
\hline hob & 2.00 & 1.70 & 1.70 & 1.30 & 3.20 & 2.30 & 4.50 & 3.00 \\
\hline hold & 9.70 & 8.30 & 7.80 & 9.00 & 7.50 & 8.30 & 8.40 & 7.60 \\
\hline hone & 2.80 & 2.00 & 3.00 & 2.30 & 2.50 & 2.10 & 2.40 & 2.20 \\
\hline hospital & 7.50 & 8.70 & 8.20 & 7.80 & 8.10 & 4.90 & 8.10 & 8.00 \\
\hline hub & 3.20 & 1.50 & 2.90 & 4.20 & 4.60 & 2.60 & 3.70 & 2.50 \\
\hline hutment & 1.70 & 1.22 & 1.50 & 1.00 & 1.90 & 2.20 & 2.10 & 2.11 \\
\hline idea & 9.20 & 8.40 & 9.00 & 8.70 & 7.60 & 8.60 & 9.00 & 7.80 \\
\hline imbibe & 2.30 & 2.40 & 2.20 & 1.50 & 6.40 & 5.70 & 7.11 & 3.20 \\
\hline impassioned & 4.30 & 4.10 & 5.10 & 4.60 & 6.40 & 6.00 & 6.10 & 5.20 \\
\hline implicit & 6.80 & 6.50 & 6.20 & 5.40 & 5.00 & 2.40 & 3.80 & 2.60 \\
\hline importance & 9.00 & 8.40 & 8.70 & 7.80 & 7.90 & 7.70 & 8.00 & 9.10 \\
\hline increase & 9.67 & 8.60 & 8.70 & 9.30 & 8.10 & 6.80 & 8.70 & 7.00 \\
\hline indignant & 4.40 & 4.10 & 4.10 & 4.40 & 4.70 & 3.60 & 5.90 & 5.20 \\
\hline infrared & 6.20 & 5.10 & 5.70 & 3.40 & 3.60 & 3.30 & 2.30 & 3.40 \\
\hline interfaith & 3.80 & 2.33 & 2.80 & 2.80 & 3.60 & 3.00 & 3.90 & 2.90 \\
\hline interim & 4.50 & 3.40 & 4.40 & 3.00 & 4.60 & 3.50 & 5.40 & 5.70 \\
\hline joss & 2.20 & 1.10 & 2.00 & 1.40 & 3.10 & 2.30 & 3.40 & 1.90 \\
\hline journalese & 1.70 & 1.90 & 2.30 & 1.10 & 2.00 & 1.00 & 1.10 & 2.30 \\
\hline kale & 2.20 & 1.30 & 1.60 & 1.30 & 7.50 & 6.10 & 7.90 & 1.40 \\
\hline kept & 7.80 & 7.50 & 6.90 & 6.20 & 8.50 & 7.10 & 8.90 & 9.10 \\
\hline kinetic & 6.00 & 5.90 & 7.00 & 5.80 & 5.30 & 3.60 & 4.90 & 3.80 \\
\hline language & 8.80 & 7.90 & 9.40 & 7.10 & 7.90 & 6.70 & 8.40 & 8.10 \\
\hline lath & 2.10 & 1.30 & 2.40 & 1.40 & 2.10 & 3.00 & 2.30 & 3.10 \\
\hline legatee & 1.60 & 1.60 & 1.40 & 1.50 & 1.60 & 2.10 & 1.90 & 2.40 \\
\hline limited & 8.40 & 8.70 & 7.30 & 7.80 & 7.60 & 5.80 & 7.90 & 8.00 \\
\hline lobar & 2.70 & 1.10 & 1.70 & 2.10 & 1.70 & 1.40 & 1.60 & 2.80 \\
\hline lore & 3.89 & 1.90 & 1.90 & 4.70 & 4.50 & 4.50 & 4.20 & 2.70 \\
\hline luminous & 6.70 & 5.40 & 4.60 & 4.00 & 2.90 & 2.60 & 3.00 & 3.30 \\
\hline lummox & 1.50 & 1.30 & 1.50 & 1.20 & 2.30 & 2.10 & 1.90 & 2.90 \\
\hline machine & 9.10 & 6.50 & 8.50 & 7.70 & 8.80 & 8.20 & 9.30 & 9.30 \\
\hline madding & 2.70 & 1.50 & 2.10 & 1.50 & 2.30 & 2.00 & 2.10 & 1.60 \\
\hline maelstrom & 2.20 & 2.60 & 1.30 & 1.70 & 2.90 & 1.60 & 3.70 & 2.20 \\
\hline mandate & 5.30 & 4.00 & 7.20 & 5.60 & 3.80 & 1.90 & 3.20 & 1.50 \\
\hline manifold & 3.60 & 4.50 & 4.00 & 2.90 & 3.20 & 1.50 & 2.70 & 1.90 \\
\hline
\end{tabular}


APPENDIX (Continued)

\begin{tabular}{|c|c|c|c|c|c|c|c|c|}
\hline \multirow[b]{2}{*}{ Stimulus Words } & \multicolumn{4}{|c|}{ Visual } & \multicolumn{4}{|c|}{ Auditory } \\
\hline & $\mathbf{R}$ & W & $\mathrm{H}$ & $\mathbf{S}$ & $\mathbf{R}$ & $\mathrm{W}$ & $\mathrm{H}$ & $\mathrm{S}$ \\
\hline manned & 4.50 & 5.30 & 5.00 & 2.20 & 2.70 & 2.40 & 4.20 & 2.10 \\
\hline mar & 2.40 & 1.80 & 2.50 & 2.70 & 3.80 & 2.30 & 5.30 & 2.30 \\
\hline mawkish & 1.30 & 1.00 & 1.20 & 1.20 & 1.40 & 2.10 & 2.00 & 4.50 \\
\hline maybe & 9.50 & 8.78 & 8.70 & 9.80 & 8.20 & 7.50 & 8.20 & 7.70 \\
\hline meaning & 8.20 & 8.30 & 8.40 & 7.80 & 8.00 & 6.60 & 8.20 & 7.90 \\
\hline meld & 3.00 & 1.20 & 3.20 & 2.80 & 2.10 & 1.30 & 1.20 & 6.60 \\
\hline member & 8.20 & 7.30 & 8.30 & 8.00 & 8.80 & 8.20 & 8.50 & 6.20 \\
\hline method & 9.20 & 8.10 & 9.10 & 6.70 & 8.50 & 6.80 & 7.90 & 8.90 \\
\hline milling & 3.00 & 2.60 & 4.10 & 3.10 & 3.10 & 2.60 & 2.00 & 1.60 \\
\hline million & 8.10 & 6.50 & 7.50 & 9.10 & 7.90 & 6.50 & 8.50 & 6.10 \\
\hline modern & 8.80 & 9.20 & 8.10 & 8.60 & 9.50 & 7.50 & 9.40 & 9.10 \\
\hline modicum & 1.30 & 1.10 & 1.60 & 1.20 & 2.00 & 1.22 & 1.90 & 1.90 \\
\hline moldboard & 1.70 & 1.40 & 1.80 & 2.60 & 3.20 & 1.80 & 2.60 & 1.70 \\
\hline monaraul & 1.60 & 1.70 & 1.30 & 1.50 & 2.00 & 1.90 & 1.70 & 1.90 \\
\hline monastic & 2.80 & 1.40 & 2.30 & 2.20 & 3.80 & 3.40 & 3.20 & 2.10 \\
\hline money & 9.40 & 8.90 & 9.70 & 9.90 & 7.60 & 7.20 & 9.10 & 7.70 \\
\hline mores & 3.50 & 2.50 & 4.40 & 3.20 & 4.10 & 2.70 & 5.20 & 3.40 \\
\hline morning & 9.40 & 7.40 & 8.60 & 9.40 & 7.00 & 7.70 & 6.90 & 6.80 \\
\hline mosque & 3.40 & 3.50 & 4.00 & 2.60 & 2.20 & 1.30 & 2.70 & 3.00 \\
\hline mot & 2.20 & 1.60 & 1.70 & 1.60 & 1.80 & 1.78 & 2.10 & 4.60 \\
\hline mutton & 1.60 & 1.90 & 3.30 & 2.00 & 3.40 & 4.20 & 3.60 & 3.30 \\
\hline myriad & 3.00 & 2.70 & 2.20 & 2.30 & 4.00 & 3.40 & 4.10 & 4.20 \\
\hline natural & 8.44 & 7.40 & 7.90 & 7.70 & 8.50 & 7.70 & 8.60 & 7.30 \\
\hline officer & 8.50 & 7.20 & 8.30 & 6.80 & 7.30 & 5.80 & 7.10 & 8.10 \\
\hline ominous & 6.30 & 3.60 & 5.80 & 3.40 & 2.10 & 1.50 & 2.10 & 1.80 \\
\hline papal & 2.00 & 2.40 & 2.30 & 2.50 & 4.60 & 3.50 & 4.20 & 2.90 \\
\hline parapet & 2.50 & 2.50 & 1.40 & 1.10 & 1.90 & 1.00 & 2.50 & 2.80 \\
\hline pariah & 1.20 & 1.30 & 1.00 & 1.10 & 1.80 & 1.50 & 1.30 & 2.00 \\
\hline parvenu & 1.67 & 1.40 & 1.80 & 1.50 & 1.80 & 1.50 & 1.70 & 1.20 \\
\hline pay & 9.00 & 8.00 & 9.50 & 8.90 & 6.50 & 7.30 & 8.10 & 7.00 \\
\hline penury & 3.10 & 1.20 & 2.30 & 1.40 & 1.20 & 1.30 & 1.80 & 1.50 \\
\hline peril & 5.20 & 3.70 & 2.50 & 3.30 & 5.20 & 3.00 & 3.40 & 3.50 \\
\hline personal & 8.50 & 7.40 & 8.70 & 8.22 & 8.50 & 6.60 & 8.80 & 9.00 \\
\hline phonemic & 3.80 & 1.80 & 2.20 & 2.20 & 1.80 & 1.20 & 1.90 & 1.00 \\
\hline piazza & 2.90 & 2.70 & 3.10 & 3.70 & 4.90 & 3.90 & 5.90 & 4.30 \\
\hline pious & 3.30 & 2.00 & 3.00 & 2.40 & 5.20 & 3.00 & 5.20 & 4.10 \\
\hline piquant & 1.30 & 1.70 & 2.20 & 1.60 & 2.40 & 2.20 & 1.60 & 3.60 \\
\hline plight & 4.60 & 3.80 & 4.70 & 2.50 & 3.20 & 2.70 & 4.20 & 3.10 \\
\hline position & 8.80 & 7.80 & 9.00 & 7.89 & 8.30 & 6.50 & 8.10 & 7.60 \\
\hline posse & 4.10 & 4.40 & 4.70 & 3.90 & 5.40 & 3.30 & 4.80 & 4.30 \\
\hline press & 8.30 & 7.20 & 7.90 & 6.78 & 8.20 & 7.40 & 8.90 & 9.50 \\
\hline price & 8.20 & 7.30 & 8.90 & 9.40 & 6.30 & 5.50 & 6.80 & 6.00 \\
\hline probably & 8.10 & 8.30 & 9.70 & 8.78 & 9.30 & 8.10 & 9.60 & 9.90 \\
\hline production & 8.20 & 8.67 & 7.40 & 7.90 & 8.50 & 6.70 & 8.80 & 7.90 \\
\hline quality & 8.10 & 8.56 & 8.50 & 7.90 & 7.90 & 6.70 & 9.50 & 8.00 \\
\hline quill & 4.10 & 2.10 & 3.30 & 2.60 & 1.90 & 1.60 & 2.00 & 1.20 \\
\hline quint & 3.00 & 1.80 & 1.90 & 1.50 & 2.40 & 1.80 & 3.70 & 4.10 \\
\hline quixotic & 1.30 & 1.40 & 1.40 & 1.20 & 1.50 & 1.80 & 1.90 & 3.70 \\
\hline radio & 8.40 & 7.40 & 8.80 & 9.11 & 8.20 & 6.50 & 7.70 & 8.20 \\
\hline reach & 8.30 & 7.60 & 7.60 & 8.00 & 9.00 & 6.90 & 8.70 & 7.90 \\
\hline reaction & 8.40 & 7.80 & 8.70 & 7.80 & 7.20 & 7.90 & 8.40 & 6.30 \\
\hline regime & 5.10 & 5.50 & 4.50 & 4.00 & 6.10 & 3.80 & 6.40 & 5.20 \\
\hline religion & 9.20 & 7.70 & 8.10 & 8.50 & 8.60 & 8.20 & 9.10 & 8.20 \\
\hline remember & 8.30 & 8.30 & 9.10 & 9.00 & 8.90 & 6.80 & 7.80 & 9.10 \\
\hline report & 8.20 & 8.90 & 8.90 & 8.20 & 8.30 & 7.40 & 8.90 & 7.60 \\
\hline retention & 5.40 & 5.30 & 7.50 & 5.50 & 4.00 & 2.60 & 4.70 & 4.20 \\
\hline rev & 3.20 & 2.30 & 3.10 & 2.50 & 3.40 & 4.00 & 5.90 & 4.50 \\
\hline rot & 7.60 & 4.70 & 5.30 & 6.90 & 3.70 & 5.10 & 4.10 & 4.20 \\
\hline rubicund & 1.78 & 1.10 & 1.40 & 1.10 & 1.40 & 1.90 & 1.50 & 2.70 \\
\hline rubric & 1.30 & 1.20 & 1.50 & 1.40 & 2.30 & 2.30 & 2.90 & 1.70 \\
\hline
\end{tabular}


APPENDIX (Continued)

\begin{tabular}{|c|c|c|c|c|c|c|c|c|}
\hline \multirow[b]{2}{*}{ Stimulus Words } & \multicolumn{4}{|c|}{ Visual } & \multicolumn{4}{|c|}{ Auditory } \\
\hline & $\mathrm{R}$ & W & $\mathbf{H}$ & $\mathbf{S}$ & $\mathbf{R}$ & W & $\mathrm{H}$ & $\mathrm{S}$ \\
\hline run & 9.80 & 7.80 & 8.90 & 9.40 & 6.40 & 6.90 & 7.50 & 7.00 \\
\hline running & 8.10 & 7.30 & 8.89 & 9.40 & 5,90 & 6.50 & 7.20 & 6.00 \\
\hline rusk & 2.30 & 3.50 & 2.70 & 2.90 & 1.60 & 2.50 & 2.00 & 1.30 \\
\hline sacrosanct & 2.10 & 1.00 & 1.80 & 1.10 & 1.70 & 2.40 & 2.70 & 4.60 \\
\hline savior & 4.10 & 4.30 & 4.90 & 2.60 & 3.80 & 3.20 & 3.10 & 2.80 \\
\hline scrim & 1.40 & 1.40 & 2.20 & 1.80 & 5.80 & 5.30 & 5.80 & 3.11 \\
\hline secular & 5.60 & 3.20 & 5.50 & 3.90 & 4.60 & 3.40 & 5.50 & 3.00 \\
\hline semantic & 4.70 & 4.00 & 4.40 & 2.70 & 3.10 & 2.60 & 5.00 & 2.90 \\
\hline sepia & 3.20 & 2.00 & 2.40 & 2.20 & 1.90 & 2.60 & 2.40 & 1.70 \\
\hline shill & 2.50 & 2.20 & 1.70 & 1.40 & 1.60 & 1.80 & 3.30 & 2.40 \\
\hline shone & 4.30 & 5.30 & 3.50 & 3.70 & 8.40 & 7.30 & 8.80 & 8.40 \\
\hline shrove & 2.20 & 1.30 & 1.50 & 1.80 & 2.40 & 2.60 & 1.90 & 1.40 \\
\hline similar & 7.90 & 7.50 & 8.10 & 8.70 & 7.20 & 6.50 & 6.90 & 6.10 \\
\hline skiff & 2.70 & 1.20 & 1.90 & 1.50 & 3.20 & 2.70 & 4.90 & 4.50 \\
\hline sluice & 2.10 & 1.60 & 2.40 & 1.00 & 1.30 & 1.00 & 2.78 & 4.20 \\
\hline sortie & 2.10 & 1.60 & 3.10 & 2.80 & 1.40 & 1.67 & 2.10 & 3.40 \\
\hline sound & 8.80 & 7.80 & 8.50 & 9.70 & 5.40 & 4.40 & 5.40 & 4.20 \\
\hline south & 8.40 & 9.10 & 8.70 & 7.90 & 8.40 & 5.50 & 8.30 & 7.40 \\
\hline space & 8.89 & 7.50 & 8.40 & 9.40 & 7.90 & 8.60 & 7.90 & 8.10 \\
\hline spate & 1.50 & 1.40 & 3.00 & 2.30 & 2.30 & 1.80 & 2.40 & 2.30 \\
\hline spurious & 1.70 & 2.00 & 2.20 & 1.60 & 1.44 & 2.50 & 1.70 & 1.90 \\
\hline squalid & 2.50 & 1.30 & 2.30 & 1.30 & 2.40 & 1.70 & 2.67 & 1.60 \\
\hline squall & 3.20 & 3.80 & 5.30 & 3.20 & 3.10 & 2.30 & 4.00 & 2.60 \\
\hline stalwart & 2.50 & 1.40 & 1.40 & 2.40 & 2.10 & 2.10 & 1.89 & 2.00 \\
\hline standard & 8.60 & 8.00 & 8.70 & 7.70 & 7.50 & 7.40 & 8.40 & 9.50 \\
\hline stay & 8.80 & 8.70 & 9.10 & 8.50 & 9.10 & 6.80 & 9.00 & 9.00 \\
\hline stein & 4.30 & 1.60 & 2.10 & 3.60 & 6.30 & 5.20 & 7.50 & 6.10 \\
\hline stiletto & 2.00 & 2.00 & 1.30 & 1.10 & 7.80 & 6.40 & 8.20 & 1.90 \\
\hline story & 8.70 & 8.00 & 9.00 & 7.30 & 8.10 & 8.90 & 8.90 & 9.40 \\
\hline street & 8.70 & 8.10 & 9.20 & 9.20 & 8.40 & 7.70 & 8.50 & 9.10 \\
\hline study & 9.10 & 8.60 & 9.40 & 9.60 & 8.20 & 7.50 & 8.10 & 6.30 \\
\hline support & 7.80 & 9.33 & 8.50 & 8.20 & 9.10 & 7.70 & 8.10 & 7.60 \\
\hline technical & 7.80 & 8.00 & 7.80 & 7.90 & 8.40 & 6.30 & 8.80 & 7.60 \\
\hline temperature & 9.20 & 7.10 & 8.60 & 8.30 & 9.20 & 9.30 & 9.50 & 8.70 \\
\hline theory & 9.20 & 8.30 & 8.90 & 6.78 & 8.00 & 5.40 & 7.80 & 7.60 \\
\hline thwack & 1.90 & 1.10 & 1.30 & 1.70 & 1.40 & 1.30 & 1.70 & 1.89 \\
\hline tilth & 1.90 & 1.30 & 1.70 & 1.30 & 1.11 & 1.80 & 2.40 & 3.20 \\
\hline tonal & 4.10 & 2.30 & 3.00 & 1.50 & 1.50 & 1.30 & 2.50 & 1.80 \\
\hline tone & 6.80 & 6.30 & 7.80 & 6.90 & 3.70 & 5.00 & 5.70 & 4.00 \\
\hline transom & 2.40 & 1.70 & 2.20 & 1.70 & 3.70 & 3.20 & 6.40 & 5.00 \\
\hline trenchant & 1.90 & 1.50 & 1.70 & 2.50 & 2.60 & 1.60 & 4.60 & 3.00 \\
\hline trial & 7.60 & 6.70 & 8.50 & 7.00 & 7.10 & 5.80 & 7.60 & 4.80 \\
\hline trig & 3.10 & 2.90 & 4.50 & 4.30 & 3.10 & 2.80 & 2.60 & 2.60 \\
\hline triptych & 2.00 & 1.40 & 1.10 & 1.20 & 4.40 & 2.60 & 2.20 & 2.22 \\
\hline truth & 7.70 & 9.20 & 8.10 & 8.80 & 7.50 & 6.30 & 8.00 & 7.40 \\
\hline understand & 8.60 & 8.30 & 9.20 & 8.70 & 2.90 & 2.20 & 2.00 & 2.00 \\
\hline vector & 3.90 & 5.70 & 5.70 & 3.60 & 6.00 & 4.10 & 6.00 & 5.30 \\
\hline vengeance & 6.30 & 5.80 & 6.30 & 3.20 & 3.30 & 2.10 & 4.10 & 3.20 \\
\hline voice & 8.10 & 6.50 & 8.40 & 8.30 & 7.70 & 6.70 & 8.40 & 6.40 \\
\hline welch & 4.00 & 3.90 & 3.60 & 2.80 & 3.00 & 3.00 & 3.60 & 4.00 \\
\hline western & 7.00 & 8.40 & 8.00 & 6.60 & 8.30 & 7.30 & 8.70 & 8.80 \\
\hline wharves & 1.80 & 1.30 & 1.30 & 1.40 & 1.60 & 1.30 & 2.56 & 2.11 \\
\hline wife & 8.30 & 7.40 & 8.40 & 6.60 & 8.20 & 6.80 & 8.00 & 6.20 \\
\hline wold & 1.20 & 1.50 & 1.50 & 1.33 & 2.50 & 2.90 & 4.50 & 3.10 \\
\hline yon & 1.80 & 1.80 & 1.60 & 1.30 & 2.50 & 1.90 & 1.78 & 2.90 \\
\hline zeal & 3.90 & 3.70 & 3.10 & 2.60 & 8.30 & 6.30 & 7.40 & 7.40 \\
\hline
\end{tabular}

(Manuscript received November I, 1996;

revision accepted for publication March 26, 1997.) 